\title{
Lateral-torsional buckling of singly symmetric web-tapered thin-walled I-beams: 1D model vs. shell FEA
}

\author{
Anísio Andrade ${ }^{\mathrm{a}}$, Dinar Camotim ${ }^{\mathrm{b}, *}$, P. Borges Dinis ${ }^{\mathrm{b}}$ \\ ${ }^{a}$ Department of Civil Engineering, University of Coimbra, Pólo II, Pinhal de Marrocos, 3030-290 Coimbra, Portugal \\ ${ }^{\mathrm{b}}$ Department of Civil Engineering, ICIST/IST, Technical University of Lisbon, Av. Rovisco Pais, 1049-001 Lisboa, Portugal
}

Received 17 November 2004; accepted 12 August 2006

Available online 10 January 2007

\begin{abstract}
This paper assesses the global performance and the underlying assumptions of a recently developed one-dimensional model characterising the elastic lateral-torsional buckling behaviour of singly symmetric tapered thin-walled open beams, which is able to account for the influence of the pre-buckling deflections. A comparative study involving the critical load factors and buckling modes yielded by (i) the one-dimensional model and (ii) two-dimensional shell finite element analyses (reference results) is presented and discussed. The results concern I-section cantilevers and simply supported beams (i) with uniform or linearly tapered webs, (ii) equal or unequal uniform flanges and (iii) acted by point loads applied at the free end or mid-span sections, respectively. In general, the one-dimensional predictions are found to agree well with the shell finite element results. Some significant discrepancies are also recorded (for the shorter beams), which are due to the occurrence of relevant cross-section distortion or localised buckling phenomena.
\end{abstract}

(C) 2006 Civil-Comp Ltd. and Elsevier Ltd. All rights reserved.

Keywords: Lateral-torsional buckling; Web-tapered I-beams; One-dimensional model; Rayleigh-Ritz method; Shell finite element analysis; Pre-buckling deflections

\section{Introduction}

Tapered members are widely used in the steel construction industry, because of their (i) structural efficiency, which in turn may lead to significant material savings, (ii) ability to meet architectural and functional requirements and (iii) competitive fabrication costs. However, a designer can only take full advantage of the benefits of beam tapering provided that he is equipped with reliable and efficient methods of analysis, which (i) lead to accurate predictions of the tapered member structural behaviour and, at the same time, (ii) do not involve a computer effort prohibitive for routine applications.

The structural behaviour of the vast majority of laterally unrestrained beams, either prismatic or tapered, is gov-

\footnotetext{
* Corresponding author. Tel.: +35121 8418403; fax: +351218497650.

E-mail address: dcamotim@civil.ist.utl.pt (D. Camotim).
}

erned by lateral-torsional buckling (LTB), a rather complex instability phenomenon combining minor axis bending and torsion. Then, it is just logical to expect that, for the sake of uniformity and ease-of-use, one attempts to develop design methodologies that are valid for both prismatic and tapered beams. In order to achieve this goal, the most "natural" approach consists of trying to modify the rules and procedures already available in the current steel design codes for prismatic beams, in order to extend their range of validity to tapered beams. Therefore, it is not surprising to learn that this was precisely the path followed in Eurocode 3 (EC3-EN or simply EC3 [1] - recall that the pre-standard EC3-ENv [2] did not include any provisions regarding the design of tapered members). However, if one wishes to extend the range of validity of the current design methodologies, it becomes necessary to develop efficient (i.e. as accurate and simple as possible) methods to evaluate the elastic critical load factor (i.e. the load factor associated with the first bifurcation point) of any given 
tapered beam, an indispensable step to determine its normalised slenderness. Concerning this issue, it is worth noting that EC3 [1] merely suggests the performance of a finite element analysis - presumably, by adopting shell or solid elements to discretise the beam.

Very recently, the first two authors proposed a onedimensional model to analyse the elastic LTB behaviour of singly symmetric tapered thin-walled open beams [3], which can be viewed as an extension of Vlassov's theory [4] (applicable to prismatic bars). Subsequently, the model was extended to account for the influence of the pre-buckling deflections $[5,6]$. The elastic critical load factors and corresponding buckling modes yielded by this model, numerically implemented by means of the Rayleigh-Ritz method [3,5], were only verified through the comparison with results reported by other authors (e.g. [7-11]), which also rely on more or less similar a priori hypotheses. Hence, it becomes necessary to put the model to the test in order to validate (or, better yet, to corroborate) both (i) the assumptions underlying the one-dimensional model and (ii) the LTB predictions provided by their adoption. One is particularly interested in obtaining evidence to either support or refute the more unexpected or even paradoxical consequences of the one-dimensional model, namely:

(i) The LTB behaviours of prismatic and tapered beams are, in general, qualitatively different. By this assertion it is meant that, in tapered beams exhibiting a certain geometrical feature (to be specified later), piecewise prismatic analyses will not converge to the correct LTB solution.

(ii) For a specific typology and loading conditions, the minimum buckling resistance is not necessarily associated with the beam having the least amount of material.

Then, the main objective of this work is to present and discuss a comparative study between the LTB results obtained through analyses based on (i) the one-dimensional model mentioned in the previous paragraph and (ii) two-dimensional shell finite element (FE) models the latter are performed in the code ABAQUs [12] and taken as reference. These results (elastic critical load factors and corresponding buckling mode shapes) concern several Isection cantilevers and simply supported beams (i) having equal or unequal uniform flanges and linearly tapered webs and (ii) subjected to point loads applied at various locations of the free end section (cantilevers) or mid-span section (simply supported beams).

Although the subject of elastic LTB of tapered thinwalled open beams is an old one, its investigation with the use of shell finite element models has not attracted the attention of many researchers in the past. Among the available studies, one should mention those due to Polyzois and Qing [13], Polyzois and Raftoyiannis [14], Ronagh and Bradford [15] and Braham and Heck [16], which focus on web-tapered I-beams and use shell elements to model the web, whereas the flanges (and also any existing transverse stiffeners) are modelled with beam elements. Notice, however, that the motivation of the present work (to assess the performance of a specific one-dimensional model) differs significantly from the purposes of the above researchers. Indeed, while (i) the first two investigations tried to evaluate the applicability of the North American code provisions that were relevant at the time, (ii) the third one is concerned with lateral-distortional buckling, an instability phenomenon characterised by simultaneous lateral deflections and in-plane cross-section deformations, and (iii) the last one addresses the numerical simulation of experimental tests recently carried out at the University of Liège (further details about the tests and the FE simulations can be found in [17]). Moreover, none of these investigations accounts for the influence of the pre-buckling deflections on the LTB behaviour of tapered beams.

\section{One-dimensional model}

The central objective of the various theories of beams, which have a long and rich history in structural mechanics, is to achieve a one-dimensional characterisation (i.e. having the parameter of a certain curve and, possibly, time as the only independent variables) of the behaviour of a particular class of bodies, having two characteristic dimensions much smaller than the third one, the length [18]. The need for tractable and accurate lower-dimensional theories is dictated by the formidable mathematical obstacles (analytical as well as numerical) posed, even today, by the threedimensional continuum models. Nevertheless, it must be stressed that beam models are intrinsically approximate, since the actual bodies one deals with are threedimensional.

The technical or engineering beam theories are typically obtained by incorporating a set of a priori assumptions into a three-dimensional continuum model, which may exhibit different levels of sophistication. These assumptions (i) are mostly concerned with the "form" of some of the unknowns, such as displacement or stress components, (ii) may be more or less realistic and (iii) can foster a greater or smaller simplification, although some inconsistencies may eventually appear. ${ }^{1}$

In the next subsections, one sketches a brief outline, reasonably self-contained, of the one-dimensional model recently developed and numerically implemented by Andrade and Camotim $[3,5,6]$, which is intended to characterise the elastic LTB behaviour of singly symmetric tapered thinwalled open beams (i.e. its goal is to provide accurate predictions of the bifurcation load factors and buckling modes). Note that a thin-walled beam exhibits an additional geometrical feature, concerning the relation between

\footnotetext{
${ }^{1}$ Perhaps the best-known illustration of this statement is provided by Bernoulli's assumption of plane sections.
} 
the two cross-section dimensions: the wall thickness is an order of magnitude smaller than the mid-line length. As far as the mechanical behaviour is concerned, a thin-walled open beam can be characterised by the fact that it resists torsion as a spatial system: the cross-sections exhibit outof-plane warping and, since this warping generally varies along the beam axis, non negligible longitudinal normal strains and stresses appear.

\subsection{Geometrical description of the undeformed beam}

Let $B$ be the region of the Euclidean three-dimensional space occupied by the beam in its undeformed configuration. It is assumed that B can be generated by the translation, along a straight-line segment of length $L$, of a plane figure (i) remaining normal to that line segment and (ii) whose shape and dimensions are allowed to vary smoothly. This generating figure is (i) simply connected, (ii) symmetric with respect to its minor central axis and (iii) may be viewed as the result of ascribing a small thickness to an open "base curve", possibly with sharp corners and branching points. Moreover, it is also assumed that the surface swept by the generating figure symmetry axis is plane. The mid-surface of B is denoted by S.

Consider a fixed orthonormal right-handed Cartesian frame $\left\{O ; \boldsymbol{e}_{1}, \boldsymbol{e}_{2}, \boldsymbol{e}_{3}\right\}$, with coordinates $\{x, y, z\}$, such that (i) $\boldsymbol{e}_{1}$ is parallel to the line segment used to generate $\mathrm{B}$ and (ii) the coordinate plane $\left\{\boldsymbol{e}_{1}, \boldsymbol{e}_{3}\right\}$ coincides with the plane swept by the minor central axis of the generating figure (i.e. $\left\{\boldsymbol{e}_{1}, \boldsymbol{e}_{3}\right\}$ is a symmetry plane of B). A beam crosssection (cross-section mid-line) is defined as the material surface (curve) having an undeformed configuration, denoted by $\mathrm{A}(x)(\mathrm{L}(x))$, that corresponds to the intersection of $B\left(\right.$ of $S$ ) by a plane perpendicular to $e_{1}{ }^{2}$ In particular, the end cross-sections are initially contained in the planes $x=0$ and $x=L$. These considerations are illustrated in Fig. 1, for the specific case of a tapered I-section beam with unequal flanges.

A material point on the beam mid-surface is identified by its coordinates $(x, \bar{y}, \bar{z})$ in the undeformed configuration: $x$ specifies the cross-section, whereas $\bar{y}$ and $\bar{z}$ define the location of the point on $\mathrm{L}(x)$ (the bar is used to indicate a quantity associated with the mid-surface). An alternative description is constructed by assigning to $\mathrm{S}$ a system of Gaussian coordinates $\left\{\theta^{1}, \theta^{2}\right\}$, such that (i) $\theta^{1}=x$ and (ii) $\theta^{2}$ measures the arc length along $\mathrm{L}\left(\theta^{1}\right)$, with the curve $\theta^{2}=0$ lying on the symmetry plane $\left\{\boldsymbol{e}_{1}, \boldsymbol{e}_{3}\right\}$. According to this alternative viewpoint, both $\bar{y}$ and $\bar{z}$ are functions of $\theta^{1}$ and $\theta^{2}$.

At this point, it is appropriate to recall some basic concepts and results from the differential geometry of surfaces (e.g. [19]). At a given (non-singular) point of S, the covar-

\footnotetext{
${ }^{2}$ The chief merit of these definitions is avoiding any reference to centroidal or shear-centre lines, which, in general, cannot be defined a priori in an unambiguous way [18].
}

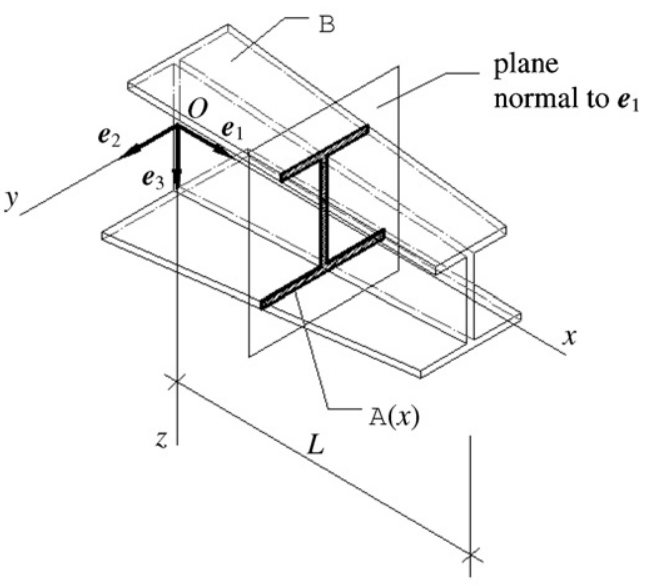

Fig. 1. Tapered I-section beam: undeformed configuration and fixed reference frame.

iant base vectors of the plane tangent to $\mathrm{S}$, associated with the Gaussian coordinate system, are defined by ${ }^{3}$

$\boldsymbol{a}_{\alpha}\left(\theta^{1}, \theta^{2}\right)=\frac{\partial \overline{\boldsymbol{r}}\left(\theta^{1}, \theta^{2}\right)}{\partial \theta^{\alpha}}=\overline{\boldsymbol{r}}_{, \alpha}\left(\theta^{1}, \theta^{2}\right)$

where $\overline{\boldsymbol{r}}\left(\theta^{1}, \theta^{2}\right)=\theta^{1} \boldsymbol{e}_{1}+\bar{y}\left(\theta^{1}, \theta^{2}\right) \boldsymbol{e}_{2}+\bar{z}\left(\theta^{1}, \theta^{2}\right) \boldsymbol{e}_{3}$ is the position vector (relative to the origin of the fixed Cartesian frame). These base vectors are tangent to the coordinate curves and, in addition, one should note that $\boldsymbol{a}_{2}\left(\theta^{1}, \theta^{2}\right)$ is a unit vector. The contravariant (or dual) basis $\left\{\boldsymbol{a}^{1}\left(\theta^{1}, \theta^{2}\right), \boldsymbol{a}^{2}\left(\theta^{1}, \theta^{2}\right)\right\}$ is defined by the relation

$\boldsymbol{a}^{\alpha}\left(\theta^{1}, \theta^{2}\right) \cdot \boldsymbol{a}_{\beta}\left(\theta^{1}, \theta^{2}\right)=\delta_{\beta}^{\alpha}$,

where the dot stands for the Euclidean inner product and $\delta_{\beta}^{\alpha}$ is the Kronecker symbol. The symmetric second-order tensor field with covariant components given by

$\boldsymbol{a}_{\alpha \beta}\left(\theta^{1}, \theta^{2}\right)=\boldsymbol{a}_{\alpha}\left(\theta^{1}, \theta^{2}\right) \cdot \boldsymbol{a}_{\beta}\left(\theta^{1}, \theta^{2}\right)$

is the metric tensor of S, also known as the first fundamental form of S. The contravariant components of this surface tensor field are

$\boldsymbol{a}^{\alpha \beta}\left(\theta^{1}, \theta^{2}\right)=\boldsymbol{a}^{\alpha}\left(\theta^{1}, \theta^{2}\right) \cdot \boldsymbol{a}^{\beta}\left(\theta^{1}, \theta^{2}\right)=\left(\boldsymbol{a}_{\alpha \beta}\left(\theta^{1}, \theta^{2}\right)\right)^{-1}$

and the area element $\mathrm{d} A\left(\theta^{1}, \theta^{2}\right)$ at $\left(\theta^{1}, \theta^{2}\right) \in \mathrm{S}$ reads

$$
\begin{aligned}
\mathrm{d} A\left(\theta^{1}, \theta^{2}\right) & =\left\|\boldsymbol{a}_{1}\left(\theta^{1}, \theta^{2}\right) \times \boldsymbol{a}_{2}\left(\theta^{1}, \theta^{2}\right)\right\| \mathrm{d} \theta^{1} \mathrm{~d} \theta^{2} \\
& =\sqrt{a\left(\theta^{1}, \theta^{2}\right)} \mathrm{d} \theta^{1} \mathrm{~d} \theta^{2},
\end{aligned}
$$

where $a\left(\theta^{1}, \theta^{2}\right)=\operatorname{det}\left(a_{\alpha \beta}\left(\theta^{1}, \theta^{2}\right)\right)$ and $\|\cdot\|$ and $\times$ denote the Euclidean norm and cross product, respectively.

In general, the base vectors $\boldsymbol{a}_{\alpha}\left(\theta^{1}, \theta^{2}\right)$ (or $\boldsymbol{a}^{\alpha}\left(\theta^{1}, \theta^{2}\right)$, for that matter) are not orthogonal. It is thus convenient to introduce orthonormal vectors $\boldsymbol{A}_{\mathrm{I}}\left(\theta^{1}, \theta^{2}\right)$ and $\boldsymbol{A}_{\mathrm{II}}\left(\theta^{1}, \theta^{2}\right)$, spanning the plane tangent to $\mathrm{S}$ and such that one has

\footnotetext{
${ }^{3}$ Unless otherwise stated, a Greek index takes values from the set $\{1,2\}$ and the summation convention is adopted.
} 
$\boldsymbol{A}_{\mathrm{II}}\left(\theta^{1}, \theta^{2}\right)=\boldsymbol{a}_{2}\left(\theta^{1}, \theta^{2}\right)$. From these conditions, one may write

$$
\boldsymbol{A}_{\mathrm{I}}\left(\theta^{1}, \theta^{2}\right)=\frac{1}{\sqrt{a\left(\theta^{1}, \theta^{2}\right)}} \boldsymbol{a}_{1}\left(\theta^{1}, \theta^{2}\right)-\frac{a_{12}\left(\theta^{1}, \theta^{2}\right)}{\sqrt{a\left(\theta^{1}, \theta^{2}\right)}} \boldsymbol{a}_{2}\left(\theta^{1}, \theta^{2}\right) .
$$

\subsection{Fundamental assumptions}

The proposed model, developed under the assumption that both the strains and the derivatives of the displacements along $e_{1}$ are small (i.e. negligible in comparison with unity), is applicable to beams (i) displaying the geometrical features described in the previous subsection, (ii) made of a St. Venant-Kirchhoff material (e.g. [20]), with Young's modulus $E$ and Poisson's ratio $v$, and (iii) subjected to the generic system of conservative loads depicted in Fig. 2, which (iii $\left.{ }_{1}\right)$ act initially on the plane of symmetry of the undeformed beam $\left(\left\{\boldsymbol{e}_{1}, \boldsymbol{e}_{3}\right\}\right)$ and $\left(\mathrm{iii}_{2}\right)$ are proportional to a single load factor $\lambda$.

The beams may exhibit either a linear or a non-linear pre-buckling equilibrium path, which amounts to saying that the influence of the pre-buckling deflections on their LTB behaviour may be neglected or not. In the latter case, it is nevertheless assumed that there exists a linear relationship between the membrane forces associated with a fundamental state and the derivatives of the generalised displacements.

The beams are regarded as membrane shells (e.g. [19]), upon which the following kinematical constraints are imposed:

(i) The projection of each cross-section mid-line on a plane perpendicular to $\boldsymbol{e}_{1}$ experiences no distortion (i.e. retains its shape and dimensions) throughout the whole deformation process.

(ii) The mid-surface shear strains (involving fibres originally oriented along vectors $\boldsymbol{A}_{\mathrm{I}}$ and $\boldsymbol{A}_{\mathrm{II}}$ ) are negligible.

The above kinematical constraints extend, to the case of tapered beams, the classical Vlassov's hypotheses [4], com-

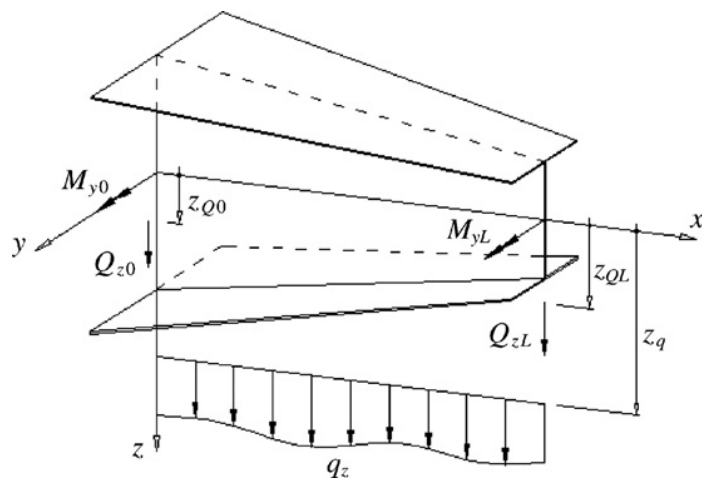

Fig. 2. External conservative loads. monly adopted in the overall buckling analysis of prismatic thin-walled open beams. It is important to stress that the first constraint inevitably implies that this model is unable to capture any local-plate, distortional or mixed instability phenomena (i.e. the beam is constrained to buckle in a "pure" global mode - LTB).

The strain energy associated with uniform torsion, which is completely disregarded in the membrane shell model, is added separately by (i) considering the expression valid for prismatic beams, but (ii) accounting for the variation of the cross-sectional property $J$ with $x$, a procedure backed by both theoretical and experimental evidence [21].

\subsection{Kinematics}

According to the first of the two kinematical constraints stated above, the transverse motion (i.e. parallel to the coordinate plane $\left.\left\{\boldsymbol{e}_{2}, \boldsymbol{e}_{3}\right\}\right)$ of any given cross-section midline is described by the laws of rigid-body kinematics. It may therefore be broken down into (i) a rotation $\Phi$ about $\boldsymbol{e}_{1}$, followed by (ii) a translation with components $V$ and $W$ along $\boldsymbol{e}_{2}$ and $\boldsymbol{e}_{3}$, respectively. The second constraint makes it possible to express the mid-line displacements along $\boldsymbol{e}_{1}$ as a function of $V, W$ and $\Phi$, to within an uniform longitudinal displacement $U$. Indeed, it is shown in $[3,5,6]$ that the mid-surface displacement field $\bar{U} \boldsymbol{e}_{1}+\bar{V} \boldsymbol{e}_{2}+\bar{W} \boldsymbol{e}_{3}$ takes on the form

$$
\begin{aligned}
\bar{U}\left(\theta^{1}, \theta^{2}\right)= & U\left(\theta^{1}\right)-\bar{y}\left(\theta^{1}, \theta^{2}\right)\left(V_{, 1}\left(\theta^{1}\right) \cos \Phi\left(\theta^{1}\right)\right. \\
& \left.+W_{, 1}\left(\theta^{1}\right) \sin \Phi\left(\theta^{1}\right)\right)-\bar{z}\left(\theta^{1}, \theta^{2}\right)\left(W_{, 1}\left(\theta^{1}\right) \cos \Phi\left(\theta^{1}\right)\right. \\
& \left.-V_{, 1}\left(\theta^{1}\right) \sin \Phi\left(\theta^{1}\right)\right)-\bar{\omega}\left(\theta^{1}, \theta^{2}\right) \Phi, 1\left(\theta^{1}\right), \\
\bar{V}\left(\theta^{1}, \theta^{2}\right)= & V\left(\theta^{1}\right)-\bar{y}\left(\theta^{1}, \theta^{2}\right)\left(1-\cos \Phi\left(\theta^{1}\right)\right) \\
& -\bar{z}\left(\theta^{1}, \theta^{2}\right) \sin \Phi\left(\theta^{1}\right), \\
\bar{W}\left(\theta^{1}, \theta^{2}\right)= & W\left(\theta^{1}\right)+\bar{y}\left(\theta^{1}, \theta^{2}\right) \sin \Phi\left(\theta^{1}\right)-\bar{z}\left(\theta^{1}, \theta^{2}\right)\left(1-\cos \Phi\left(\theta^{1}\right)\right),
\end{aligned}
$$

where $U, V, W$ and $\Phi$ are the generalised displacements,

$\bar{\omega}\left(\theta^{1}, \theta^{2}\right)=\int_{\mathrm{C}\left(\theta^{1}, \theta^{2}\right)}\left(\bar{y}\left(\theta^{1}, s\right) \bar{z}_{, s}\left(\theta^{1}, s\right)-\bar{z}\left(\theta^{1}, s\right) \bar{y}_{, s}\left(\theta^{1}, s\right)\right) \mathrm{d} s$

and $\mathrm{C}\left(\theta^{1}, \theta^{2}\right)$ is the segment of $\mathrm{L}\left(\theta^{1}\right)$ comprised between the point defined by $\theta^{2}=0$ and the point under consideration, defined by the Gaussian coordinates $\left(\theta^{1}, \theta^{2}\right)$. Note that the restriction of $\bar{\omega}$ to $\mathrm{L}\left(\theta^{1}\right)$ represents a sectorial coordinate.

It is also shown in Refs. [3,5,6] that the covariant components of the Green-St. Venant membrane strain tensor (i.e. half the change of metric tensor associated with the above displacement field) read $^{4}$

\footnotetext{
${ }^{4}$ In order to simplify the notation, the independent variables of a function will henceforth be given explicitly only when this is needed for special emphasis.
} 


$$
\begin{aligned}
\bar{E}_{11}= & U_{, 1}-\bar{y}\left(V_{, 11} \cos \Phi+W_{, 11} \sin \Phi\right) \\
& -\bar{z}\left(W_{, 11} \cos \Phi-V_{, 11} \sin \Phi\right)-\bar{\omega} \Phi_{, 11}-\psi \Phi_{, 1} \\
& +\frac{1}{2}\left(V_{, 1}^{2}+W_{, 1}^{2}+\left(\bar{y}^{2}+\bar{z}^{2}\right) \Phi_{, 1}^{2}\right), \\
\bar{E}_{12}= & \bar{E}_{21}=0, \\
\bar{E}_{22}= & \frac{1}{2}\left(\bar{y}_{, 2}\left(V_{, 1} \cos \Phi+W_{, 1} \sin \Phi-\bar{z} \Phi_{, 1}\right)\right. \\
& \left.+\bar{z}_{, 2}\left(W_{, 1} \cos \Phi-V_{, 1} \sin \Phi+\bar{y} \Phi_{, 1}\right)\right)^{2},
\end{aligned}
$$

where the function

$\psi=\bar{\omega}_{, 1}+\bar{y}_{, 1} \bar{z}-\bar{z}_{, 1} \bar{y}$

stems directly from the cross-section variation (one has $\psi=0$ in prismatic beams).

\subsection{Total potential energy}

The total potential energy $\Pi$ of the beam-load system consists of the beam (elastic) strain energy $U$ and the potential energy of the external loads $\mathrm{V}_{\mathrm{e}}$. As mentioned before, the beam strain energy is the sum of two separate contributions: (i) the membrane strain energy and (ii) the uniform torsion strain energy. The former is a quadratic and positive definite functional in terms of the Green-St. Venant membrane strain tensor [19]:

$\frac{1}{2} \int_{\mathrm{S}} t a^{\alpha \beta \gamma \delta} \bar{E}_{\chi \delta} \bar{E}_{\alpha \beta} \mathrm{d} A=\frac{1}{2} \int_{\mathrm{S}} \bar{n}^{\alpha \beta} \bar{E}_{\alpha \beta} \mathrm{d} A$,

where (i) the contravariant components of the two-dimensional elasticity tensor of the membrane shell are given by

$a^{\alpha \beta \gamma \delta}=E\left(v a^{\alpha \beta} a^{\chi \delta}+\frac{1}{2}(1-v)\left(a^{\alpha \chi} a^{\beta \delta}+a^{\alpha \delta} a^{\beta \chi}\right)\right)$

(note that the approximation $1-v^{2} \approx 1$, often used in the context of beam theories - e.g. [4,22], was adopted), (ii) the contravariant components of the membrane force (or stress resultant) tensor read

$\bar{n}^{\alpha \beta}=\operatorname{ta}^{\alpha \beta \gamma \delta} \bar{E}_{\chi \delta}$

(iii) $\mathrm{d} A$ is the area element on $\mathrm{S}$, given by Eq. (5), and (iv) $t$ is the wall thickness. By introducing the kinematical relations (11)-(13) into Eq. (15), one can write the membrane strain energy in terms of the generalised displacements. As for the part of the strain energy associated with uniform (or St. Venant) torsion, it is simply given by

$\frac{E}{4(1+v)} \int_{0}^{L} J \Phi_{, 1}^{2} \mathrm{~d} \theta^{1}$

where $J\left(\theta^{1}\right)=4 \int_{\mathrm{A}\left(\theta^{1}\right)} n^{2} \mathrm{~d} y \mathrm{~d} z$ and $n\left(\theta^{1}, y, z\right)$ measures the distance from the points in $A\left(\theta^{1}\right)$ to $\mathrm{L}\left(\theta^{1}\right)$.

Finally, with reference to Fig. 2, the potential energy of the external loads is defined by

$$
\begin{aligned}
\mathrm{V}_{\mathrm{e}}= & -\int_{0}^{L} q_{z}\left(W-z_{q}(1-\cos \Phi)\right) \mathrm{d} \theta^{1} \\
& -Q_{z 0}\left(W(0)-z_{Q 0}(1-\cos \Phi(0))\right) \\
& -Q_{z L}\left(W(L)-z_{Q L}(1-\cos \Phi(L))\right) \\
& +M_{y 0}\left(W_{, 1}(0) \cos \Phi(0)-V_{, 1}(0) \sin \Phi(0)\right) \\
& +M_{y L}\left(W_{, 1}(L) \cos \Phi(L)-V_{, 1}(L) \sin \Phi(L)\right) .
\end{aligned}
$$

\subsection{Bifurcation analysis}

According to Trefftz's criterion (e.g. [23]), a bifurcation point on the fundamental equilibrium path (parameterised by $\lambda$ ) is identified by the variational equation

$\delta\left(\delta^{2} \Pi\right)=0$,

which states the stationarity of the second variation of $\Pi$ from a given fundamental state in the class of kinematically admissible displacement variations. If one neglects the contribution of the membrane forces $\bar{n}^{22}$ (one of the basic assumptions of elementary beam theory), the following expression for $\delta^{2} \Pi$ is obtained [5] (see also [6], where this expression has been specialised for doubly symmetric beams):

$$
\begin{aligned}
\delta^{2} \Pi= & \frac{E}{2} \int_{0}^{L}\left(A^{*}\left(u_{, 1}+W_{, 1}^{\mathrm{f}} w_{, 1}\right)^{2}-2 S_{y}^{*}\left(u_{, 1}+W_{, 1}^{\mathrm{f}} w_{, 1}\right) w_{, 11}\right. \\
& +I_{y}^{*} w_{, 11}^{2}+I_{z}^{*}\left(v_{, 11}+W_{, 11}^{\mathrm{f}} \phi\right)^{2}+I_{\omega}^{*} \phi_{, 11}^{2}+I_{\psi}^{*} \phi_{, 1}^{2} \\
& +2 I_{\omega z}^{*}\left(v_{, 11}+W_{, 11}^{\mathrm{f}} \phi\right) \phi_{, 11}+2 I_{\psi z}^{*}\left(v_{, 11}+W_{, 11}^{\mathrm{f}} \phi\right) \phi_{, 1} \\
& \left.+2 I_{\omega \psi}^{*} \phi_{, 1} \phi_{, 11}\right) \mathrm{d} \theta^{1}+\frac{G}{2} \int_{0}^{L} J \phi_{, 1}^{2} \mathrm{~d} \theta^{1} \\
& +\frac{1}{2} \int_{0}^{L} M_{y}^{\mathrm{f}}\left(2 v_{, 11} \phi+W_{, 11}^{\mathrm{f}} \phi^{2}+\frac{z_{G}^{*} I_{\rho}^{*}-\beta_{y}^{*} I_{y}^{*}}{z_{G}^{*} S_{y}^{*}-I_{y}^{*}} \phi_{, 1}^{2}\right) \mathrm{d} \theta^{1} \\
& +\frac{z_{q}}{2} \int_{0}^{L} q_{z} \phi^{2} \mathrm{~d} \theta^{1}+\frac{z_{Q 0}}{2} Q_{z 0} \phi(0)^{2}+\frac{z_{Q L}}{2} Q_{z L} \phi(L)^{2} \\
& -\frac{M_{y 0}}{2}\left(2 v_{, 1}(0) \phi(0)+W_{, 1}^{\mathrm{f}}(0, \lambda) \phi(0)^{2}\right) \\
& -\frac{M_{y L}}{2}\left(W_{, 1}^{\mathrm{f}}(L, \lambda) \phi(L)^{2}+2 v_{, 1}(L) \phi(L)\right),
\end{aligned}
$$

where (i) $u, v, w$ and $\phi$ are kinematically admissible variations of the generalised displacements (independent from $\lambda$ ), (ii) the functions

$A^{*}=\int_{\mathrm{L}\left(\theta^{1}\right)} t^{*} \mathrm{~d} \theta^{2}, \quad S_{y}^{*}=\int_{\mathrm{I}\left(\theta^{1}\right)} \bar{z} t^{*} \mathrm{~d} \theta^{2}, \quad I_{y}^{*}=\int_{\mathrm{I}\left(\theta^{1}\right)} \bar{z}^{2} t^{*} \mathrm{~d} \theta^{2}$,

$I_{z}^{*}=\int_{\mathrm{I}\left(\theta^{1}\right)} \bar{y}^{2} t^{*} \mathrm{~d} \theta^{2}, \quad I_{\omega}^{*}=\int_{\mathrm{I}\left(\theta^{1}\right)} \bar{\omega}^{2} t^{*} \mathrm{~d} \theta^{2}, \quad I_{\psi}^{*}=\int_{\mathrm{I}\left(\theta^{1}\right)} \psi^{2} t^{*} \mathrm{~d} \theta^{2}$,

$I_{\omega z}^{*}=\int_{\mathrm{I}\left(\theta^{1}\right)} \bar{\omega} \bar{y} t^{*} \mathrm{~d} \theta^{2}, \quad I_{\psi z}^{*}=\int_{\mathrm{I}\left(\theta^{1}\right)} \psi \bar{y} t^{*} \mathrm{~d} \theta^{2}, \quad I_{\omega \psi}^{*}=\int_{\mathrm{I}\left(\theta^{1}\right)} \bar{\omega} \psi t^{*} \mathrm{~d} \theta^{2}$,

$I_{\rho}^{*}=\int_{\mathrm{I}\left(\theta^{1}\right)}\left(\bar{y}^{2}+\bar{z}^{2}\right) t^{*} \mathrm{~d} \theta^{2}, \quad \beta_{y}^{*}=\frac{1}{I_{y}^{*}} \int_{\mathrm{I}\left(\theta^{1}\right)}\left(\bar{y}^{2}+\bar{z}^{2}\right) \bar{z} t^{*} \mathrm{~d} \theta^{2}, \quad z_{G}^{*}=\frac{S_{y}^{*}}{A^{*}}$, 
with $t^{*}=t a^{-3 / 2}$, are geometrical properties of the undeformed beam, (iii) $W^{\mathrm{f}}\left(\theta^{1}, \lambda\right)$ denotes the pre-buckling deflections and (iv) $M_{y}^{\mathrm{f}}$ is the bending moment distribution in the fundamental state. Note that the derivation of Eq. (21) uses (i) the orthogonality conditions

$$
\begin{array}{lll}
\int_{\mathrm{I}\left(\theta^{1}\right)} \bar{y} t^{*} \mathrm{~d} \theta^{2}=0 & \int_{\mathrm{L}\left(\theta^{1}\right)} \bar{\omega} t^{*} \mathrm{~d} \theta^{2}=0 & \int_{\mathrm{I}\left(\theta^{1}\right)} \psi t^{*} \mathrm{~d} \theta^{2}=0 \\
\int_{\mathrm{I}\left(\theta^{1}\right)} \bar{y} \bar{z} t^{*} \mathrm{~d} \theta^{2}=0 & \int_{\mathrm{I}\left(\theta^{1}\right)} \bar{\omega} \bar{z} t^{*} \mathrm{~d} \theta^{2}=0 & \int_{\mathrm{I}\left(\theta^{1}\right)} \psi \bar{z} t^{*} \mathrm{~d} \theta^{2}=0
\end{array}
$$

and also (ii) the fact that one has, in a fundamental state,

$\int_{\mathrm{L}\left(\theta^{1}\right)}\left(a^{11}\right)^{-1 / 2}\left(\bar{n}^{11} \boldsymbol{a}_{1}+\bar{n}^{12} \boldsymbol{a}_{2}\right) \cdot \boldsymbol{e}_{1} \mathrm{~d} \theta^{2}=0$.

From a mathematical viewpoint, Eq. (20) is the variational statement of a non-linear eigenvalue problem. The non-linear character stems from the terms appearing in Eq. (21) that involve the pre-buckling deflections $W^{f}$. The removal of these terms corresponds to linearising the eigenvalue problem - linear buckling analysis - and, physically, amounts to disregarding the influence of the pre-buckling deflections on the bifurcation load factors and buckling modes (i.e. the beam is assumed to remain straight until the onset of buckling).

One of the most distinctive features of this one-dimensional model (and therefore one that should be put to the test) is the indication of qualitative differences between the LTB behaviours of prismatic and tapered beams whenever the latter exhibit $\psi \neq 0$ (this is always the case in webtapered I-beams). Indeed, the terms

$\frac{E}{2} \int_{0}^{L}\left(I_{\psi} \phi_{, 1}^{2}+2 I_{\psi z}\left(v_{, 11}+W_{, 11}^{\mathrm{f}} \phi\right) \phi_{, 1}+2 I_{\omega \psi} \phi_{, 1} \phi_{, 11}\right) \mathrm{d} \theta^{1}$

in Eq. (21) clearly show that modelling a tapered beam as an assembly of prismatic segments constitutes a conceptually incorrect procedure whenever $\psi \neq 0$, regardless of the number of segments considered. Failing to include these terms in the analysis can lead to significant errors in the critical moment predictions for tapered beams, either on the safe or unsafe side, as shown by the numerical examples presented and discussed in Refs. [3,5]. In web-tapered I-beams, it is possible to provide a simple physical explanation for these qualitative differences: due to the flange slopes, the bimoments generated during buckling have an axial resultant, which is responsible for an additional contribution to the total torque - furthermore, web tapering has an effect on the amount of warping of the flanges, since it reduces their distance from the torsion centre (in other words, web tapering constitutes, in itself, a warping constraint).

Finally, it is worth mentioning that, in the case of cantilevers or simply supported beams acted by transverse loads (all the numerical examples presented in this paper concern this type of beams), (i) $u$ and $w$ play no part whatsoever in buckling analyses and (ii) $v_{, 11}$ and $\phi$ are not independent indeed, they are related by the equation

$v_{, 11}=-\frac{1}{E I_{z}^{*}}\left(M_{y}^{\mathrm{f}}\left(1-\frac{I_{z}^{*}}{I_{y}^{*}}\right) \phi+E I_{\omega z}^{*} \phi_{, 11}+E I_{\psi z}^{*} \phi_{, 1}\right)$

or, if one neglects the influence of the pre-buckling deflections (linear buckling analysis), simply by

$v_{, 11}=-\frac{1}{E I_{z}^{*}}\left(M_{y}^{\mathrm{f}} \phi+E I_{\omega z}^{*} \phi_{, 11}+E I_{\psi z}^{*} \phi_{, 1}\right)$

(since the proof of this assertion is entirely analogous to the ones presented, albeit in more restrictive contexts, in $[3,6]$, it is omitted here). Then, one may rewrite $\delta^{2} \Pi$ exclusively in terms of the single unknown field $\phi$.

\subsection{Numerical implementation}

The one-dimensional continuum model just described was discretised by means of the Rayleigh-Ritz method (e.g. [24]), using the following approximations for the torsional rotation field $\phi\left(\theta^{1}\right)$ :

(I) Cantilevers (fully built-in at the support, taken here as corresponding to $\theta^{1}=0$ )

$\phi_{n}=\sum_{k=1}^{n} a_{k}^{(n)}\left(1-\cos \left(\frac{(2 k-1) \pi}{2 L} \theta^{1}\right)\right)$.

(II) Simply-supported beams (with the so-called "fork conditions" at both end sections)

$\phi_{n}=\sum_{k=1}^{n} a_{k}^{(n)} \sin \left(\frac{k \pi}{L} \theta^{1}\right)$.

This approximation procedure replaces the continuum model by a discrete one, with $n$ degrees of freedom - number of coordinate functions used in the approximation.

\section{Shell finite element modelling}

This section addresses the most relevant procedures involved in the LTB analysis of thin-walled open beams by the FEM (i) using the commercial code ABaqus [12] and (ii) adopting shell finite elements to model the beam behaviour. In particular, the issues discussed include (i) the adequate discretisation of the beams, (ii) the modelling of the end support conditions, (iii) the numerical technique employed to solve the eigenvalue problem arising in the linear buckling analyses (when the pre-buckling deflections are neglected) and (iv) the approach devised to perform the non-linear buckling analyses (when the pre-buckling deflections are taken into account). At this point, it is worth mentioning that the FE model may include rigid transverse web stiffeners placed at one or more cross-sections (always including the ones where a point load is applied). The presence of these stiffeners is intended to restrain (as much as possible) cross-section distortion, thus providing a better simulation of the one-dimensional model assumptions. 


\subsection{Beam discretisation}

In order to be able to determine the "exact" buckling behaviour of a thin-walled beam, it is indispensable to take into account the so-called "local deformation effects", i.e. the in-plane deformation of the beam cross-sections (e.g. [25]). These effects can be adequately incorporated in the buckling analysis if the beam is modelled by means of $2 \mathrm{D}$ shell finite elements. In this work, it was decided to use 4-node isoparametric shell elements, a choice that was dictated by (i) a slightly easier automatic generation of the finite element mesh and (ii) the fact that, in web-tapered I-beams, the longitudinal edges of the web mid-surface are not parallel. On the other hand, a previous investigation [26], carried out in the context of the local and global buckling behaviours of prismatic cold-formed steel members, has shown that, among the various shell elements with these characteristics available in the ABAQus library, the S4 element (which accounts for transverse shear deformations) is particularly adequate to perform thin-walled member buckling analyses. For the above reasons, all the cantilevers and simply supported beams analysed in this work have been modelled by means of S4 shell element meshes.

The number of degrees of freedom and integration points involved in a FEA considerably influences both the required computational effort and the accuracy of the results obtained. Preliminary convergence studies have shown that sufficiently accurate results (critical load factors and buckling modes) are obtained when the finite elements exhibit the following characteristics: (i) maximum width of about $10 \mathrm{~mm}$ at the beam largest cross-section (always the one under the largest bending moment, in the tapered cases) and (ii) equal length, corresponding to an aspect ratio (length-to-maximum width) of about 2 at the largest cross-section. Moreover, in order to make the automatic mesh generation easier, the number of finite elements was kept constant in all beam cross-sections - thus, the finite element width is variable in tapered webs. Figs. 4, 6, 9, 11 and 15, which include the FEM-based critical buckling mode shapes of some of the beams analysed, make it possible to visualise the finite element meshes employed.

To check the validity of the assumptions upon which the one-dimensional formulation is based, some FE solutions were obtained for cantilevers and simply supported beams with rigid transverse web stiffeners at one or more crosssections. In order to model the presence of these stiffeners, several rigid beam finite elements (elements RB3D2, in the ABAQUS notation) were connected to the web shell element node at the cross-sections under consideration.

\subsection{End support conditions}

The beams analysed in this work exhibit three types of end support conditions: (i) a built-in end section, (ii) a free end section and (iii) a simply supported end section with "fork conditions". The first two can be modelled in a straightforward and unambiguous fashion, either by (i) imposing that all displacements and rotations are null (built-in end section) or by (ii) letting all of them free (free end section). The modelling of a simply supported end section is a bit trickier, in order to enforce the "fork conditions" assumed by the one-dimensional model. This was achieved by considering (i) free longitudinal (warping) displacements, (ii) null transverse (membrane and flexural) displacements and (iii) free flexural rotations along the whole cross-section mid-line - note that, by preventing the transverse displacements of the cross-section mid-line, its distortion is automatically precluded. Moreover, in order to eliminate the axial rigid-body mode, the longitudinal displacement was prevented at one node of the midspan cross-section.

\subsection{Linear buckling analysis - solution of the eigenvalue problem}

Carrying out a linear buckling analysis implies the need to solve a (linear) eigenvalue problem, defined by the (discretised) beam elastic stiffness and geometric matrices. In particular, one is interested in the lowest positive eigenvalue (critical load factor $\lambda_{\text {cr }}$ ) and the corresponding eigenvector (buckling mode shape). The code ABAQus uses the subspace iteration method (e.g. [27]), also known as the inverse block power method in the applied mathematics literature. Oddly enough, it was found that, due to some yet unexplained reason, it is essential to solve the problem for the first two eigenpairs. Failing to do so may lead to a wrong computation of the critical load factor [26].

\subsection{Pre-buckling deflections}

Since the code ABAqus does not offer the possibility of performing directly non-linear buckling analyses, it was necessary to devise a "trial-and-error" strategy to account for the influence of the pre-buckling deflections. It involves the following steps:

(i) To perform a linear buckling analysis of the beam, thus determining its critical load factor $\lambda_{\text {cr.0 }}$ (which does not account for the influence of the pre-buckling deflections).

(ii) To perform a linear (first-order) analysis of the beam, acted by the load associated with $\lambda_{\text {cr.0 }}$, and to record the corresponding deformed configuration.

(iii) To perform a linear buckling analysis of a "shallow arch" (possibly an inverted one) having the shape of the deformed configuration obtained in the previous step. One obtains the (linear) critical load factor of this shallow arch $\lambda_{\text {cr.1 }}\left(>\lambda_{\text {cr. } 0}\right)$, which provides a better approximation of the "straight beam" non-linear critical load.

(iv) On the basis of the $\lambda_{\text {cr.0 }}$ and $\lambda_{\text {cr. } 1}$ values, to adopt a trial-and-error approach to find an accurate estimate of the non-linear critical load factor, which must ren- 
der compatible the solutions of both $\left(\mathrm{iv}_{1}\right)$ the straight beam first-order analysis and $\left(\mathrm{iv}_{2}\right)$ the shallow arch linear buckling analysis.

\section{One-dimensional model vs. shell FEA}

The aim of this section is twofold: (i) to assess the global performance of the one-dimensional LTB model described in Section 2 and, in the process, (ii) to investigate the validity of the a priori assumption stating that the cross-section mid-lines experience no distortion. In order to achieve these goals, a comparison is made between the LTB results provided by (i) the numerical implementation of the aforementioned one-dimensional model and (ii) ABAQus shell FEA (which are taken as reference). Moreover, the FEMbased buckling modes are inspected to detect whether cross-section distortion takes place and, if this is the case, some of the FEA are performed again, using a modified model that includes one or more rigid transverse web stiffeners. This comparative study involves the LTB analysis of several I-section cantilevers and simply supported beams, both prismatic and web-tapered, acted by point loads applied at the free end (cantilevers) or at mid-span (simply supported beams). The load factor is identified with the load itself (i.e. $\lambda \equiv Q$ ).

In Section 4.1, the linear LTB behaviour of two sets of prismatic cantilevers is addressed. These cantilevers display (i) equal or unequal flanges (i.e. doubly or singly symmetric cross-section) and (ii) the geometrical data shown in Fig. 3a. The lengths vary between 2.0 and $10.0 \mathrm{~m}$, and the tip load is applied at the intersection of the web and top flange mid-lines.

In Section 4.2, which deals with web-tapered cantilevers, the geometry of the built-in section is kept unchanged and the web height is made to vary linearly along the length so that, at the free end section, the height $h$ (measured between the flange mid-lines) is reduced to half (i.e. one always has $\alpha=h_{\min } / h_{\max }=0.5$ ). The web height linear variation is achieved in two different ways, namely by (i) keeping the top flange horizontal (i.e. parallel to the plane $\left\{\boldsymbol{e}_{1}, \boldsymbol{e}_{2}\right\}$, as shown in Fig. 3b) or (ii) having flanges with equal slopes in relation to $\left\{\boldsymbol{e}_{1}, \boldsymbol{e}_{2}\right\}$ (see Fig. $3 \mathrm{c}$ ). This gives rise to four distinct sets of web-tapered cantilevers, which combine flanges with equal or unequal (i) width and (ii) slope. As before, the lengths range from 2.0 to $10.0 \mathrm{~m}$ and the tip load acts at the intersection of the web and top flange mid-lines.

In all the cases described so far, the results presented consist of (i) linear critical loads, obtained by means of $\left(i_{1}\right)$ onedimensional analyses $\left(Q_{\mathrm{cr}}^{1 \mathrm{D}}\right)$ and $\left(\mathrm{i}_{2}\right)$ two-dimensional shell FEA ( $Q_{\mathrm{cr}}^{\mathrm{FEM}}-$ reference results) and (ii) the free end section web distortion exhibited by the FEM-based critical buckling mode. In the latter case, the aim is to assess the deviation of the "true" deformed shape of the web, in relation to the one due to a rigid body rotation parallel to the $\left\{\boldsymbol{e}_{1}, \boldsymbol{e}_{2}\right\}$ plane. For the shorter beams, reference is also made to $Q_{\mathrm{cr}}^{\mathrm{FEM}}$ values obtained in the presence of transverse web stiffeners, intended to prevent the above web distortion.
Section 4.2 closes with the linear buckling analysis of the doubly symmetric web-tapered cantilever shown in Fig. 3d, which has a tapering ratio $\alpha=h_{\min } / h_{\max }=0.2$. The point load is applied at three different locations of the free end section, namely at (i) the centroid and (ii) the intersections of the web and (top and bottom) flange mid-lines. A comparison is made between (i) the linear critical loads $Q_{\mathrm{cr}}^{\mathrm{lD}}$ and $Q_{\mathrm{cr}}^{\mathrm{FEM}}$ and also (ii) the associated critical buckling mode shapes, expressed in terms of the torsional rotation $\phi .{ }^{5}$

Next, Section 4.3 concerns the linear LTB behaviour of doubly symmetric prismatic and web-tapered simply supported beams. The beams analysed (i) display the geometrical and material data shown in Fig. 3e, (ii) have lengths varying from $6.0 \mathrm{~m}$ to $12.0 \mathrm{~m}$ and (iii) are subjected to point loads acting at the mid-span cross-section (centroid or intersection of the web and top flange mid-lines). In the web-tapered beams, the geometry of the mid-span section is kept unchanged and the web height is made to decrease linearly and identically towards both ends, with different slopes: at the supports, the height $h$ (measured between the flange mid-lines) is reduced by $40 \%, 50 \%$, $60 \%$ and $80 \%$ of its mid-span value. Thus, the beams analysed exhibit $\alpha=h_{\min } / h_{\max }$ values ranging from 0.4 to 1.0 (prismatic case).

Finally, the influence of the pre-buckling deflections on the elastic critical loads of the two sets of cantilevers depicted in Fig. $3 \mathrm{f}$ is investigated in Section 4.4. All these cantilevers, either prismatic $(\alpha=1.0)$ or web-tapered $(\alpha=0.5)$, are doubly symmetric and have lengths varying between 3.0 and $8.0 \mathrm{~m}$. The load is applied at the free end section centroid, top flange and bottom flange (or, to be precise, at the flange and web mid-line intersections). The purpose of these analyses is (i) to assess the accuracy of the estimates yielded by the one-dimensional model and (ii) to study how this accuracy is affected by such factors as the location of the applied load and the cantilever length (which also controls the flange slope in the tapered cases).

\subsection{Linear LTB behaviour of prismatic cantilevers}

Table 1 provides (i) the linear critical loads $Q_{\mathrm{cr}}^{\mathrm{FEM}}$ and $Q_{\mathrm{cr}}^{1 \mathrm{D}}$ obtained for the equal and unequal-flanged prismatic cantilevers depicted in Fig. 3a (top flange loading) and (ii) the relative errors $\Delta$ associated with the $Q_{\mathrm{cr}}^{1 \mathrm{D}}$ values, which are given by the expression $\Delta=\left(Q_{\mathrm{cr}}^{\mathrm{ID}}-Q_{\mathrm{cr}}^{\mathrm{FEM}}\right) / Q_{\mathrm{cr}}^{\mathrm{FEM}} \times 100 \%$. Moreover, Fig. 4a displays a graphic representation of the variation of $\Delta$ with $L$ for both cases. This set of LTB results leads to the following remarks:

(i) The FEM-based critical loads are always smaller than the ones obtained in a one-dimensional analysis (i.e. $\Delta>0$ ). This is due to the fact that the one-dimen-

\footnotetext{
${ }^{5}$ In the FEM-based buckling mode shapes, the torsional rotation $\phi$ is assumed to be equal to the rotation of the tension (top) flange, which is not "masked" by any cross-section in-plane deformations.
} 


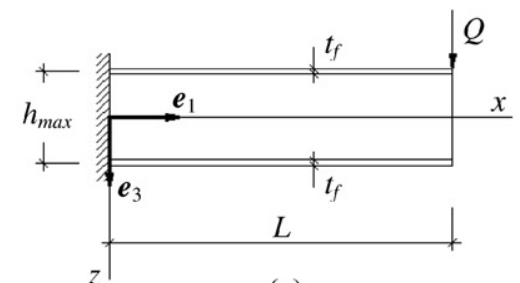

(a)

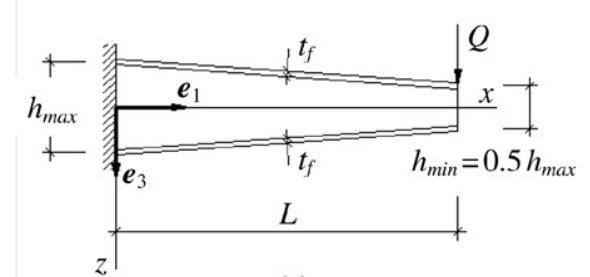

(c)

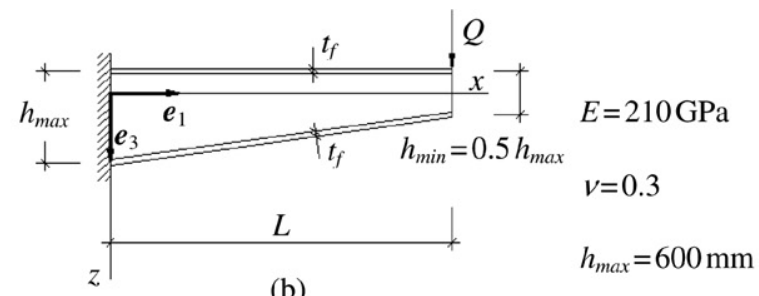

(b)
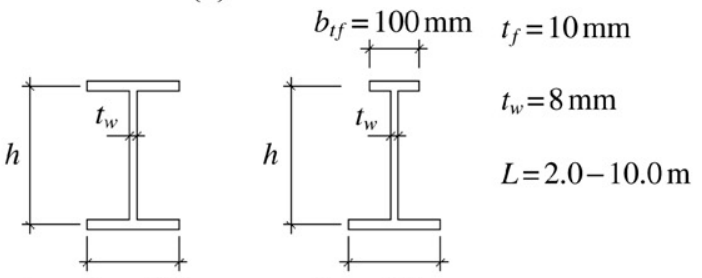

$b_{b f}=b_{t f}=180 \mathrm{~mm}$

$b_{b f}=180 \mathrm{~mm}$

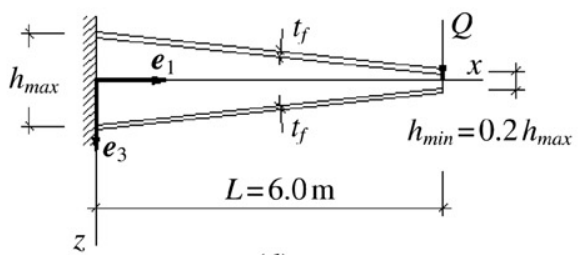

(d)

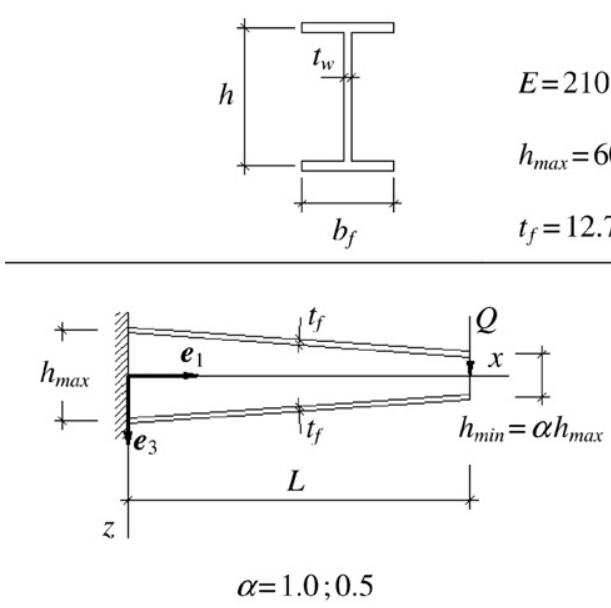

(f)

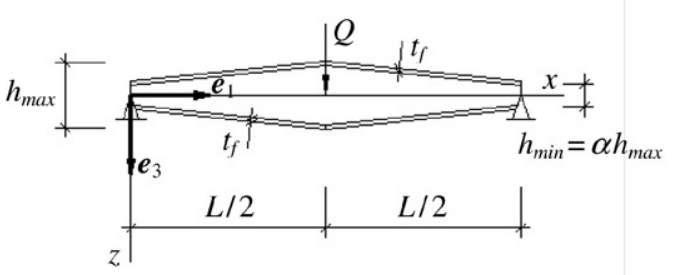

$L=6.0 \mathrm{~m} ; 9.0 \mathrm{~m} ; 12.0 \mathrm{~m} \quad \alpha=1.0-0.4$

(e)

Fig. 3. I-section cantilevers and simply supported beams: geometry, loading and material data.

Table 1

Prismatic cantilevers (Fig. 3a): linear critical loads and relative errors

\begin{tabular}{|c|c|c|c|c|c|c|}
\hline \multirow[t]{2}{*}{$L(\mathrm{~m})$} & \multicolumn{3}{|c|}{ Equal flanges } & \multicolumn{3}{|c|}{ Unequal flanges } \\
\hline & $Q_{\mathrm{cr}}^{\mathrm{FEM}}(\mathrm{kN})$ & $Q_{\mathrm{cr}}^{1 \mathrm{D}}(\mathrm{kN})$ & $\Delta(\%)$ & $Q_{\mathrm{cr}}^{\mathrm{FEM}}(\mathrm{kN})$ & $Q_{\mathrm{cr}}^{1 \mathrm{D}}(\mathrm{kN})$ & $\Delta(\%)$ \\
\hline 2.0 & 163.06 & 253.90 & 55.71 & 72.46 & 79.70 & 10.37 \\
\hline 4.0 & 42.01 & 43.68 & 3.97 & 18.52 & 19.12 & 3.21 \\
\hline 6.0 & 18.05 & 18.46 & 2.26 & 9.63 & 9.86 & 2.36 \\
\hline 8.0 & 10.57 & 10.75 & 1.67 & 6.29 & 6.40 & 1.79 \\
\hline 10.0 & 7.14 & 7.23 & 1.25 & 4.52 & 4.58 & 1.35 \\
\hline
\end{tabular}

sional model (broadly speaking, a kinematically constrained membrane shell) is stiffer than the shell finite element one. (ii) For $L \geqslant 4.0 \mathrm{~m}$, the relative errors are small $(\Delta<4 \%)$ and, moreover, they progressively decrease with $L$. As shown in Fig. 5, the distortion taking place at 


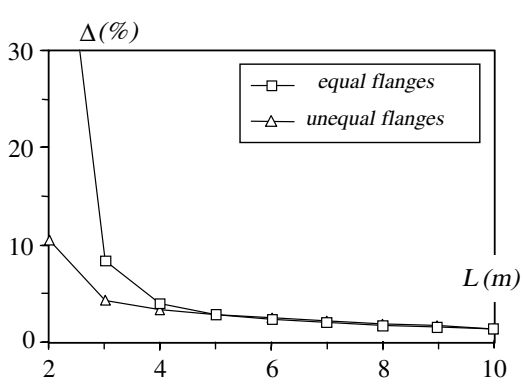

(a)

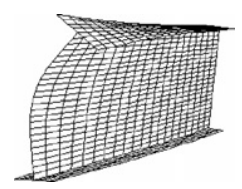

$\left(\mathrm{b}_{1}\right)$

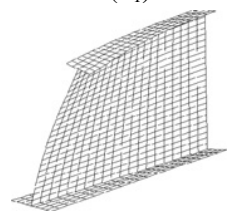

$\left(b_{3}\right)$

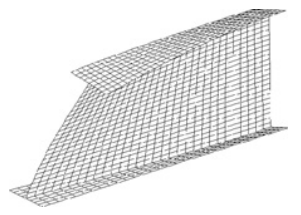

$\left(b_{2}\right)$

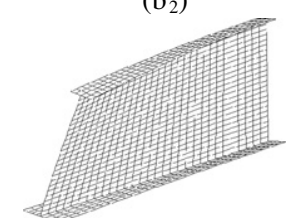

$\left(\mathrm{b}_{4}\right)$

Fig. 4. Prismatic cantilevers (Fig. 3a): (a) variation of $\Delta$ with $L$ and (b) FEM-based critical modes for $\left(\mathrm{b}_{1}\right)$ equal flanges $+L=2.0 \mathrm{~m}$, $\left(\mathrm{b}_{2}\right)$ equal flanges $+L=3.0 \mathrm{~m},\left(\mathrm{~b}_{3}\right)$ unequal flanges $+L=2.0 \mathrm{~m}$ and $\left(\mathrm{b}_{4}\right)$ unequal flanges $+L=3.0 \mathrm{~m}$.

the free end section web is not significant in this length range and, like $\Delta$, it gradually diminishes with $L$.

(iii) For lengths below $4.0 \mathrm{~m}$, both $\Delta$ and the web distortion at the free end section are substantial (particularly in the equal-flanged case). In fact, the instability phenomenon governing the behaviour of these shorter cantilevers, which exhibit an unusually large $h / L$ ratio, is not so much $\mathrm{LTB}$, but a localised buckling of the web, in the vicinity of the load point of application. This phenomenon is clearly visible in Fig. $4 b_{1}$ to $b_{4}$ (mainly in the first one), which show the critical buckling mode shapes of the shorter cantilevers provided by the ABAQUS FEM analyses, and cannot be captured by the one-dimensional model (recall that this model constrains the beam to buckle in a "pure" global mode).

(iv) In order to confirm the relevance of the above localised buckling phenomena for the shorter cantilevers ( $L=2.0 \mathrm{~m}$ and $L=3.0 \mathrm{~m}$ ), additional ABAQus FEA were performed, with rigid transverse web stiffeners located at five equally spaced cross-sections (including the free end one). The results obtained virtually coincide with the ones yielded by the one-dimensional model, both for the beams with equal and unequal flanges - the maximum of the four relative errors is less than $0.5 \%$.

\subsection{Linear LTB behaviour of web-tapered cantilevers}

Table 2 gives the values of $Q_{\mathrm{cr}}^{\mathrm{FEM}}, Q_{\mathrm{cr}}^{1 \mathrm{D}}$ and $\Delta$ pertaining to the cantilevers displayed in Fig. $3 \mathrm{~b}$ (again top flange loading), which have (i) equal or unequal uniform flanges, (ii) linearly tapered webs $(\alpha=0.5)$ and (iii) horizontal top flanges. As in the prismatic case, the FEM-based critical loads are always smaller than the ones yielded by the onedimensional model. The comparison between the results given in Tables 1 and 2 shows that, except for very short lengths, the tapered cantilever critical loads are invariably larger than those of their prismatic counterparts, even if the latter contain more material. This surprising (even paradoxical) fact, clearly visible in both the FEM and 1D results, is due to the combination of two opposing effects, both stemming from the web height decrease due to tapering [3]: (i) a material reduction (lower critical load) and (ii) a smaller distance from the load point of application to the cross-section center of rotation (higher critical load). For most of the cantilevers analysed, the latter effect dominates.

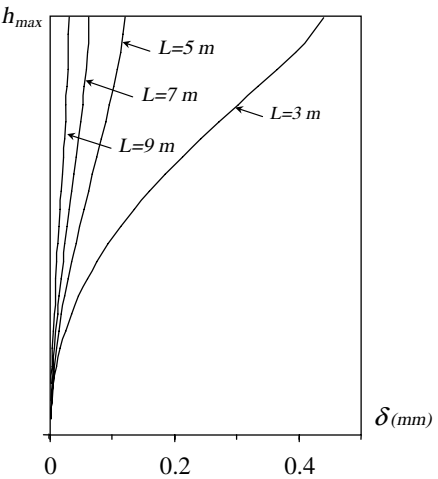

(a)

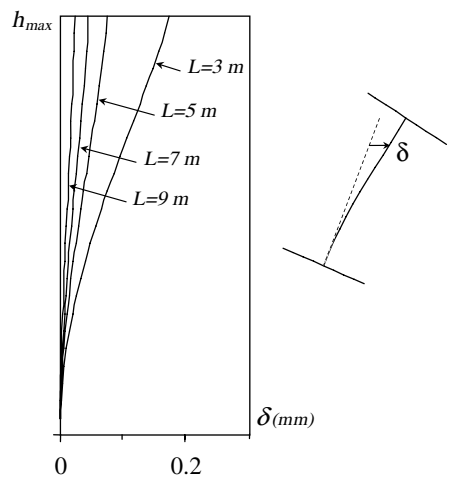

(b)

Fig. 5. Prismatic cantilevers (Fig. 3a): web distortion at the free end section for (a) equal and (b) unequal flanges - normalised FEM-based critical modes. 
Table 2

Web-tapered cantilevers (Fig. 3b): linear critical loads and relative errors

\begin{tabular}{|c|c|c|c|c|c|c|}
\hline \multirow[t]{2}{*}{$L(\mathrm{~m})$} & \multicolumn{3}{|c|}{ Equal flanges } & \multicolumn{3}{|c|}{ Unequal flanges } \\
\hline & $Q_{\mathrm{cr}}^{\mathrm{FEM}}(\mathrm{kN})$ & $Q_{\mathrm{cr}}^{1 \mathrm{D}}(\mathrm{kN})$ & $\Delta(\%)$ & $Q_{\mathrm{cr}}^{\mathrm{FEM}}(\mathrm{kN})$ & $Q_{\mathrm{cr}}^{1 \mathrm{D}}(\mathrm{kN})$ & $\Delta(\%)$ \\
\hline 2.0 & 151.91 & 195.81 & 28.90 & 68.44 & 85.05 & 24.27 \\
\hline 4.0 & 42.30 & 47.46 & 12.18 & 25.38 & 28.03 & 10.47 \\
\hline 6.0 & 22.06 & 23.46 & 6.34 & 14.66 & 15.47 & 5.55 \\
\hline 8.0 & 13.75 & 14.21 & 3.32 & 9.54 & 9.83 & 3.04 \\
\hline 10.0 & 9.24 & 9.41 & 1.77 & 6.56 & 6.68 & 1.71 \\
\hline
\end{tabular}

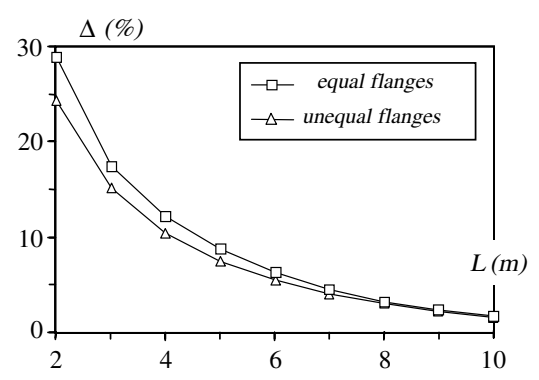

(a)

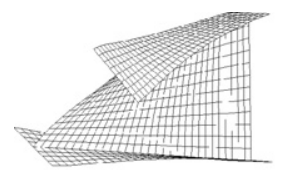

$\left(b_{1}\right)$

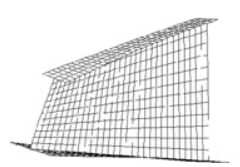

$\left(b_{3}\right)$

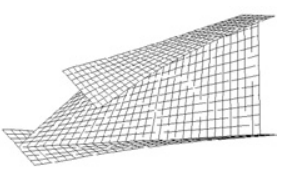

$\left(b_{2}\right)$

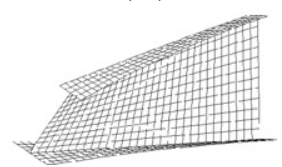

$\left(b_{4}\right)$

Fig. 6. Web-tapered cantilevers (Fig. 3b): (a) variation of $\Delta$ with $L$ and (b) FEM-based critical modes for $\left(b_{1}\right)$ equal flanges $+L=2.0 \mathrm{~m},\left(\mathrm{~b}_{2}\right)$ equal flanges $+L=3.0 \mathrm{~m},\left(\mathrm{~b}_{3}\right)$ unequal flanges $+L=2.0 \mathrm{~m}$ and $\left(\mathrm{b}_{4}\right)$ unequal flanges $+L=3.0 \mathrm{~m}$.

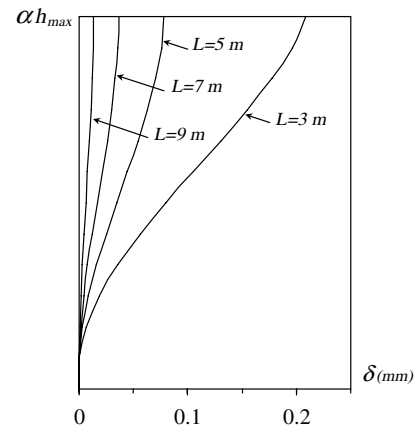

(a)

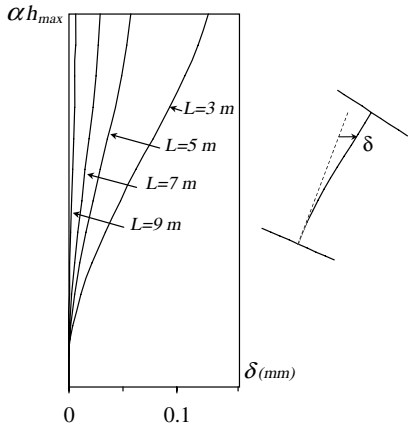

(b)

Fig. 7. Web-tapered cantilevers (Fig. 3b): web distortion at the free end section for (a) equal and (b) unequal flanges - normalised FEM-based critical modes.

Figs. $6 a$ and 7 show the variations of (i) the relative error $\Delta$ and (ii) the web distortion at the free end section with the cantilever length $L$. Their observation prompts the following comments:

(i) Both $\Delta$ and the end section web distortion increase gradually as the cantilever length diminishes. Since this increase becomes more pronounced for cantilevers shorter than about $4.0-5.0 \mathrm{~m}$, it seems fair to say that this is a "validity limit" for the one-dimensional model (i.e. it ceases to yield reasonably accurate results).

(ii) No localised web buckling phenomena is visible near the free end section (where the point load acts), even for the shortest cantilevers (see Fig. $6 b_{1}$ to $b_{4}$ ). Most

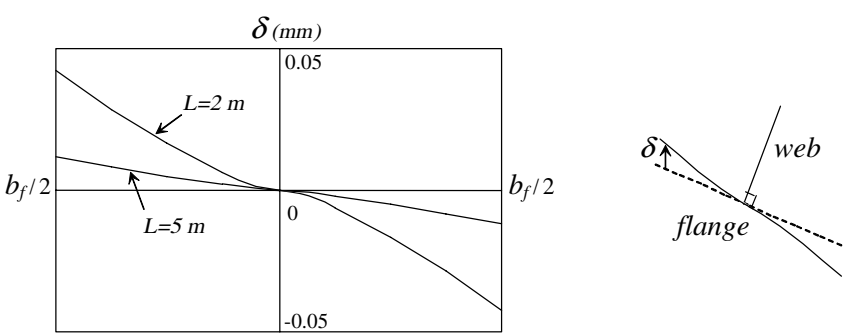

Fig. 8. Web-tapered cantilevers (Fig. 3b): compression flange distortion at the free end section for $L=2.0 \mathrm{~m}$ and $L=5.0 \mathrm{~m}$ (equal flanges) "web-stiffened" normalised FEM-based critical modes.

likely, this is due to the fact that the free end web height has been halved.

(iii) The equal-flanged cantilevers always exhibit higher $\Delta$ values and more pronounced web distortions than their unequal-flanged counterparts. However, the differences are relatively minor.

(iv) As before, the relevance of the web distortion was confirmed by the performance of additional ABAQUS FEA, concerning cantilevers with lengths $L=2.0 \mathrm{~m}$ and $L=5.0 \mathrm{~m}$ and rigid transverse web stiffeners located at several cross-sections (including the free end one). Surprisingly, it was not possible to reduce the relative errors $\Delta$ by more than about $50 \%$ (both for the beams with equal and unequal flanges), even when a significant number of stiffeners were added. These discrepancies appear to be due to the distortion of the compressed flange. Indeed, Fig. 8, which concerns the cantilevers with lengths $L=2.0 \mathrm{~m}$ and 
Table 3

Web-tapered cantilevers (Fig. 3c): linear critical loads and relative errors

\begin{tabular}{|c|c|c|c|c|c|c|}
\hline \multirow[t]{2}{*}{$L(\mathrm{~m})$} & \multicolumn{3}{|c|}{ Equal flanges } & \multicolumn{3}{|c|}{ Unequal flanges } \\
\hline & $Q_{\mathrm{cr}}^{\mathrm{FEM}}(\mathrm{kN})$ & $Q_{\mathrm{cr}}^{1 \mathrm{D}}(\mathrm{kN})$ & $\Delta(\%)$ & $Q_{\mathrm{cr}}^{\mathrm{FEM}}(\mathrm{kN})$ & $Q_{\mathrm{cr}}^{1 \mathrm{D}}(\mathrm{kN})$ & $\Delta(\%)$ \\
\hline 2.0 & 156.84 & 195.78 & 24.83 & 70.50 & 83.37 & 19.66 \\
\hline 6.0 & 22.15 & 23.45 & 5.88 & 14.73 & 15.47 & 5.01 \\
\hline 8.0 & 13.79 & 14.21 & 3.06 & 9.57 & 9.83 & 2.74 \\
\hline 10.0 & 9.26 & 9.41 & 1.59 & 6.73 & 6.73 & 0.00 \\
\hline
\end{tabular}

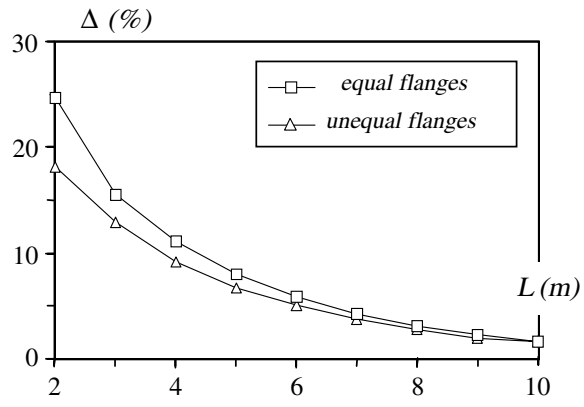

(a)

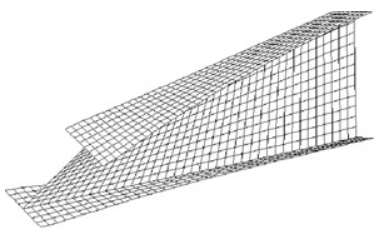

$\left(b_{1}\right)$

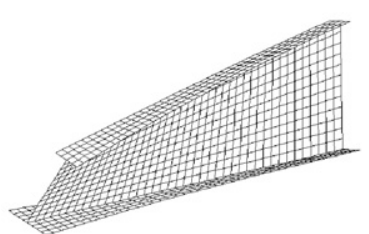

$\left(b_{2}\right)$

Fig. 9. Web-tapered cantilevers (Fig. 3c): (a) variation of $\Delta$ with $L$ and (b) FEM-based critical modes for $\left(b_{1}\right)$ equal and $\left(b_{2}\right)$ unequal flanges $(L=4.0$ m).

\section{$L=5.0 \mathrm{~m}$ and equal flanges, confirms that such a dis-} tortion takes place. ${ }^{6}$

Next, Table 3 and Fig. 9 display the results concerning web-tapered cantilevers $(\alpha=0.5)$ with both flanges equally inclined in relation to the plane $\left\{\boldsymbol{e}_{1}, \boldsymbol{e}_{2}\right\}$. Because these results are very similar to the ones obtained in the previous case (web-tapered cantilevers with horizontal top flanges), all the remarks prompted by the observation of Figs. 6 and 7 remain valid - note that the consideration of a large number of web stiffeners in the FE simulations (again for $L=2.0 \mathrm{~m}$ and $L=5.0 \mathrm{~m}$ and cantilevers with equal or unequal flanges) did not reduce the relative errors by more than about $50 \%$ (as before, the remaining differences appear to be due the compression flange distortion).

Finally, one addresses the linear LTB of the doubly symmetric web-tapered cantilever shown in Fig. 3d, which is (i) very severely tapered $(\alpha=0.2)$ and (ii) subjected to tip loads applied at the top flange, centroid and bottom flange. Besides comparing the critical loads $Q_{\mathrm{cr}}^{\mathrm{FEM}}$ and $Q_{\mathrm{cr}}^{1 \mathrm{D}}$ (see Table 4), one also assesses the accuracy of the buckling mode shapes yielded by the one-dimensional model, which are expressed in terms of the torsional rotation field $\phi$. To achieve this, Fig. 10 shows a comparison between (i) the 1D critical buckling mode shapes and (ii) FEM-based diagrams providing, in several cross-sections along the cantile-

\footnotetext{
${ }^{6}$ Note that, in this schematic representation, all the compressed flange deformed configurations have zero slope at the point of intersection with the web (they are all normal to the stiffened web).
}

Table 4

Web-tapered cantilever (Fig. 3d): linear critical loads and relative errors

\begin{tabular}{llll}
\hline Load application & $Q_{\mathrm{cr}}^{\mathrm{FEM}}(\mathrm{kN})$ & $Q_{\mathrm{cr}}^{1 \mathrm{D}}(\mathrm{kN})$ & $\Delta(\%)$ \\
\hline Top flange & 38.31 & 38.70 & 1.02 \\
Centroid & 44.36 & 44.40 & 0.09 \\
Bottom flange & 48.40 & 48.50 & 0.21 \\
\hline
\end{tabular}

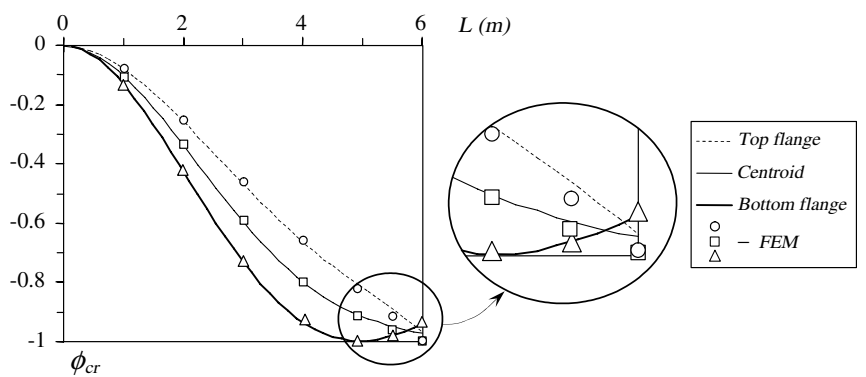

Fig. 10. Web-tapered cantilevers (Fig. 3d): 1D and FEM normalised critical modes $\left(\phi_{\mathrm{cr}}\right)$.

ver length, the rigid-body rotation of the top (tension) flange. ${ }^{7}$

\footnotetext{
${ }^{7}$ Recall that it has been assumed that, due to the virtual absence of distortion, the tension (top) flange rotation provides an accurate measure of the FEM-based cross-section rigid-body torsional rotation. Therefore, it may be directly compared with the critical buckling mode shape $\phi_{\mathrm{cr}}$ yielded by the 1D model.
} 
After observing the results presented in Table 4 and Fig. 9, one may conclude that:

(i) There is always an excellent agreement between the linear critical loads of the 1D and shell finite element models. The largest relative error occurs when the tip load is applied at the top flange, most likely because of some (very minor) local effects stemming from the transversal compression of the web induced by the tip load - such local effects cannot be captured by the one-dimensional analysis.

(ii) The critical buckling mode shapes (expressed in terms of the torsional rotation $\phi$ ) provided by the $1 \mathrm{D}$ and shell FE models practically coincide. Indeed, one only sees very small differences, near the free end section, when the load acts either at the cross-section centroid or at the top flange. Most likely, these differences are also due to the minor local effects already mentioned in the previous item.

\subsection{Linear LTB behaviour of web-tapered simply supported beams}

Table 5 gives the values of $Q_{\mathrm{cr}}^{\mathrm{FEM}}, Q_{\mathrm{cr}}^{1 \mathrm{D}}$ and $\Delta$ obtained for the simply supported beams displayed in Fig. 3e, which have (i) two longitudinal planes of symmetry (equal flanges and symmetric tapering slopes), (ii) different tapering ratios $\alpha=h_{\min } / h_{\max }$ (ranging from 0.4 to 1.0 , the latter corresponding to the prismatic case) and (iii) are acted by a mid-span point load applied at (iii ${ }_{1}$ ) the web mid-height (centroid) or ( $\mathrm{iii}_{2}$ ) the intersection of the web and top flange mid-lines. Figs. 11a and b show the variation of $Q_{\text {cr }}$ and $\Delta$ with $\alpha$, for beams having lengths $L=6.0 \mathrm{~m}$ and $L=9.0 \mathrm{~m}$. Note that Fig. 11a also includes (dashed lines) the results yielded by the one-dimensional model when the condition $\psi=0$ is imposed (i.e. when the terms given by Eq. (25) are omitted from $\left.\delta^{2} \Pi\right)$ - these same results would be obtained by using a fine enough mesh of prismatic beam FE (incorporating Vlassov's assumptions, of course).
Finally, Fig. 11c displays two FEM-based buckling mode shapes and Fig. 12 provides the variation of the web distortion at the mid-span section with the beam length $L$, for $\alpha=0.4$ and $\alpha=1.0$ (centroid and top flange loading). The observation of these LTB results leads to the following remarks:

(i) Regardless of the $\alpha$ value, both the relative error $\Delta$ and the mid-span section web distortion increase gradually as the beam length diminishes. However, note that $\left(i_{1}\right)$ errors exceeding $5 \%$ only occur for the shorter beams $(L=6.0 \mathrm{~m})$, particularly for low tapering ratios $(\alpha<0.6)$, and $\left(\mathrm{i}_{2}\right)$ all the errors concerning the critical loads of the longer beams $(L=12.0 \mathrm{~m})$ are below $3 \%$.

(ii) In the beams loaded at the intersection of the web and top flange mid-lines, the value of $\Delta$ continually decreases with $\alpha$ (see Fig. 11b). However, this statement does not remain true when the load is applied at the centroid: in this case, there is a consistent (though slight) error increase between $\alpha=0.8$ and $\alpha=1.0$. This unexpected phenomenon is probably due to an increased relevance of the web distortion - notice that the prismatic beam has the most slender web and the highest critical loads.

(iii) As shown in Fig. 11a, the shell FEA confirm the qualitative differences between the LTB behaviours of prismatic and tapered I-beams, as predicted by the one-dimensional model. Furthermore, these differences are more significant for the shorter beams $(L=6.0 \mathrm{~m})$, i.e. the ones exhibiting larger web-tapering slopes.

(iv) In order to assess the relevance of the web distortion, which is clearly visible in Fig. 12, additional Abaqus FEA were performed for the five shorter beams $(L=6.0 \mathrm{~m})$. A large number of transverse web stiffeners were included and it was found that the reduction of the relative error $\Delta$ (with respect to the corresponding unstiffened beams) increases with $\alpha$ :

Table 5

Simply supported beams (Fig. 3e): linear critical loads and relative errors

\begin{tabular}{|c|c|c|c|c|c|c|c|}
\hline \multirow[t]{2}{*}{$L(\mathrm{~m})$} & \multirow[t]{2}{*}{$\alpha$} & \multicolumn{3}{|c|}{ Top flange loading } & \multicolumn{3}{|c|}{ Centroidal loading } \\
\hline & & $Q_{\mathrm{cr}}^{\mathrm{FEM}}(\mathrm{kN})$ & $Q_{\mathrm{cr}}^{1 \mathrm{D}}(\mathrm{kN})$ & $\Delta(\%)$ & $Q_{\mathrm{cr}}^{\mathrm{FEM}}(\mathrm{kN})$ & $Q_{\mathrm{cr}}^{1 \mathrm{D}}(\mathrm{kN})$ & $\Delta(\%)$ \\
\hline \multirow[t]{3}{*}{6.0} & 0.4 & 59.28 & 65.84 & 11.07 & 100.74 & 109.75 & 8.94 \\
\hline & 0.8 & 83.28 & 85.07 & 2.15 & 126.73 & 133.26 & 5.15 \\
\hline & 1.0 & 99.30 & 100.75 & 1.46 & 141.27 & 151.28 & 7.08 \\
\hline & 0.8 & 35.65 & 36.06 & 1.15 & 50.42 & 51.31 & 1.77 \\
\hline & 1.0 & 39.93 & 40.24 & 0.78 & 54.78 & 55.99 & 2.21 \\
\hline \multirow[t]{4}{*}{12.0} & 0.4 & 17.73 & 18.22 & 2.76 & 24.18 & 24.67 & 2.03 \\
\hline & 0.6 & 18.75 & 19.03 & 1.49 & 25.32 & 25.61 & 1.15 \\
\hline & 0.8 & 20.20 & 20.36 & 0.79 & 26.87 & 27.10 & 0.86 \\
\hline & 1.0 & 21.92 & 22.03 & 0.50 & 28.67 & 28.94 & 0.94 \\
\hline
\end{tabular}



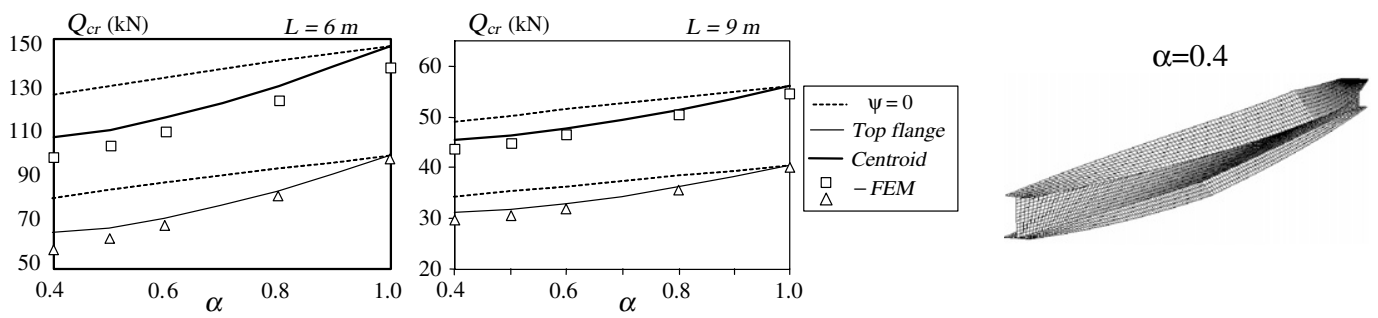

(a)
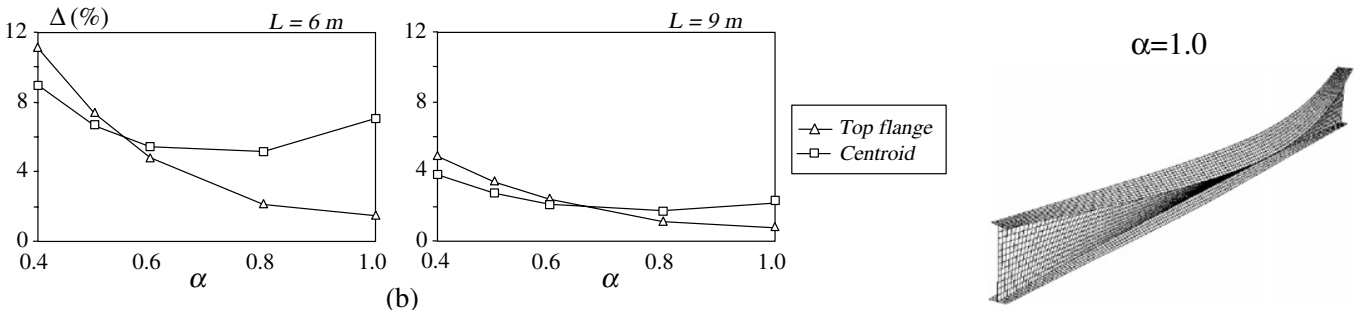

(c)

Fig. 11. Web-tapered simply supported beams (Fig. 3e): (a) variation of $Q_{\text {cr }}$ with $\alpha(L=6.0 \mathrm{~m}$ and $L=9.0 \mathrm{~m})$, (b) variation of $\Delta$ with $\alpha(L=6.0 \mathrm{~m}$ and $L=9.0 \mathrm{~m})$ and (c) FEM-based critical modes for $L=6.0 \mathrm{~m}(\alpha=0.4$ and $\alpha=1.0$ - top flange loading).

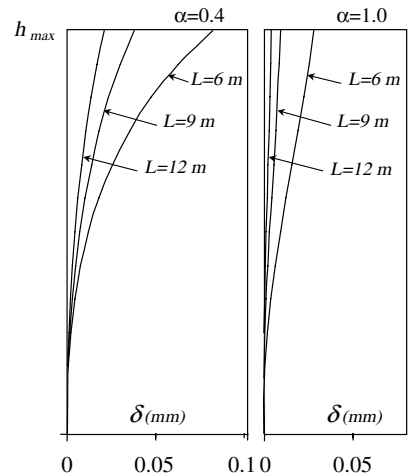

(a)

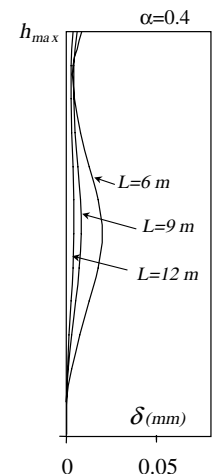

(b)

Fig. 12. Web-tapered simply supported beams (Fig. 3e): web distortion at the mid-span section for loads applied at the (a) top flange ( $\alpha=0.4$ and $\alpha=1.0)$ and (b) centroid $(\alpha=0.4$ and $\alpha=1.0)$ - normalised FEM-based critical modes.

indeed, this reduction varies continuously between (i) practically $100 \%$ (the $1 \mathrm{D}$ and FEM-based results virtually coincide), for $\alpha=1.0$, and (ii) about $35 \%$, for $\alpha=0.4$. Once again, these discrepancies can be attributed to the distortion of the compressed (top) flange, as illustrated in Fig. $13(L=6.0 \mathrm{~m}$ and load applied at the top flange) - one observes that the compressed flange distortion (i) is virtually null for $\alpha=1.0$ and (ii) progressively grows as $\alpha$ decreases. $^{8}$

\subsection{Influence of the pre-buckling deflections}

In this Section, the influence of the pre-buckling deflections on the critical loads of prismatic and webtapered cantilevers is addressed. In particular, one wishes

\footnotetext{
${ }^{8}$ Note once more that the three compressed flange deformed configurations have zero slope at the point of intersection with the (stiffened) web.
}
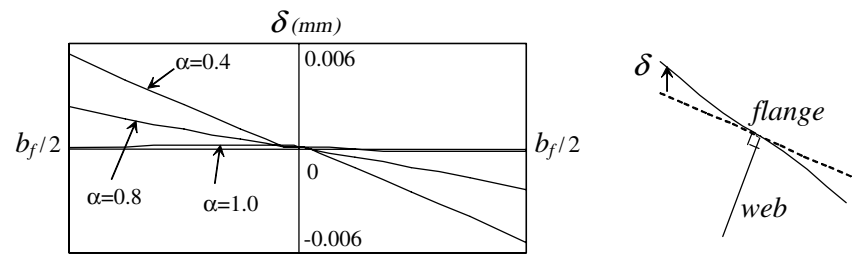

Fig. 13. Web-tapered simply supported beams (Fig. 3e): compression flange distortion at the mid-span section for $L=6.0 \mathrm{~m}$ (top flange loading and $\alpha=0.4 ; 0.8 ; 1.0)$ - "web-stiffened" normalised FEM-based critical modes.

to assess how accurately the one-dimensional model can capture this influence. In order to achieve this goal, one analyses the LTB behaviour of the web-tapered $(\alpha=0.5)$ and prismatic $(\alpha=1.0)$ cantilevers shown in Fig. 3f. Tables 6 and 7 provide, for each web-tapered and prismatic cantilever, four critical load values, which were obtained from (i) linear (i.e. with the pre-buckling deflections neglected) 
Table 6

Web-tapered cantilevers (Fig. 3f $-\alpha=0.5$ ): linear and non-linear critical loads $(\mathrm{kN})$

\begin{tabular}{|c|c|c|c|c|c|c|c|c|c|c|c|c|}
\hline \multirow[t]{2}{*}{$L(\mathrm{~m})$} & \multicolumn{4}{|c|}{ Top flange } & \multicolumn{4}{|c|}{ Centroid } & \multicolumn{4}{|c|}{ Bottom flange } \\
\hline & $Q_{\text {cr.lin }}^{1 \mathrm{D}}$ & $Q_{\text {cr.n1 }}^{1 \mathrm{D}}$ & $Q_{\text {cr.lin }}^{\mathrm{FEM}}$ & $Q_{\text {cr.nl }}^{\mathrm{FEM}}$ & $Q_{\text {cr.lin }}^{1 \mathrm{D}}$ & $Q_{\mathrm{cr} . \mathrm{nl}}^{1 \mathrm{D}}$ & $Q_{\text {cr.lin }}^{\mathrm{FEM}}$ & $Q_{\text {cr.nl }}^{\mathrm{FEM}}$ & $Q_{\text {cr.lin }}^{1 \mathrm{D}}$ & $Q_{\mathrm{cr} . \mathrm{nl}}^{1 \mathrm{D}}$ & $Q_{\mathrm{cr} . \mathrm{Iin}}^{\mathrm{FEM}}$ & $Q_{\mathrm{cr} . \mathrm{nl}}^{\mathrm{FEM}}$ \\
\hline 4.0 & 34.0 & 35.2 & 31.6 & 32.7 & 56.6 & 61.2 & 53.5 & 57.6 & 70.4 & 76.4 & 70.0 & 76.1 \\
\hline 6.0 & 15.5 & 16.3 & 15.2 & 15.9 & 20.5 & 22.1 & 20.3 & 21.8 & 23.8 & 25.7 & 23.7 & 25.7 \\
\hline 8.0 & 8.5 & 9.0 & 8.4 & 9.0 & 10.2 & 11.0 & 10.2 & 10.9 & 11.4 & 12.3 & 11.4 & 12.3 \\
\hline
\end{tabular}

Table 7

Prismatic cantilevers (Fig. 3f $-\alpha=1$ ): linear and non-linear critical loads $(\mathrm{kN})$

\begin{tabular}{|c|c|c|c|c|c|c|c|c|c|c|c|c|}
\hline \multirow[t]{2}{*}{$L(\mathrm{~m})$} & \multicolumn{4}{|c|}{ Top flange } & \multicolumn{4}{|c|}{ Centroid } & \multicolumn{4}{|c|}{ Bottom flange } \\
\hline & $Q_{\mathrm{cr} . \operatorname{lin}}^{1 \mathrm{D}}$ & $Q_{\mathrm{cr} . \mathrm{nl}}^{1 \mathrm{D}}$ & $Q_{\mathrm{cr} \cdot \mathrm{lin}}^{\mathrm{FEM}}$ & $Q_{\mathrm{cr} . n \mathrm{l}}^{\mathrm{FEM}}$ & $Q_{\text {cr.lin }}^{1 \mathrm{D}}$ & $Q_{\mathrm{cr} . \mathrm{nl}}^{1 \mathrm{D}}$ & $Q_{\mathrm{cr} \cdot \operatorname{lin}}^{\mathrm{FEM}}$ & $Q_{\mathrm{cr} . n 1}^{\mathrm{FEM}}$ & $Q_{\text {cr.lin }}^{1 \mathrm{D}}$ & $Q_{\mathrm{cr} . \mathrm{nl}}^{1 \mathrm{D}}$ & $Q_{\text {cr.lin }}^{\mathrm{FEM}}$ & $Q_{\text {cr.nl }}^{\mathrm{FEM}}$ \\
\hline 4.0 & 24.1 & 24.3 & 22.8 & 23.0 & 58.8 & 61.0 & 47.4 & 48.6 & 84.5 & 88.0 & 83.4 & 87.2 \\
\hline
\end{tabular}

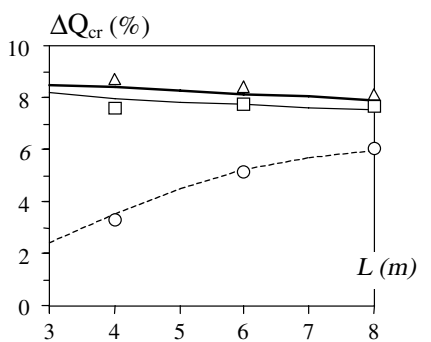

(a)
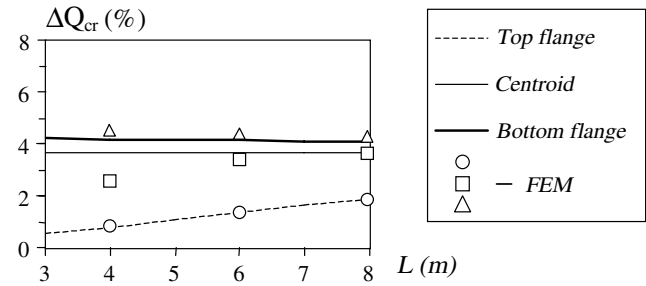

(b)

Fig. 14. Influence of the pre-buckling deflections on $Q_{\mathrm{cr}}$ : variation of the percentage increase $\Delta Q_{\mathrm{cr}}$ with $L$ for (a) web-tapered and (b) prismatic cantilevers (Fig. 3f).

or non-linear LTB analyses performed by means of the (ii) one-dimensional or shell FE models. They are designated as follows: (i) $Q_{\mathrm{cr} . l i n}^{1 \mathrm{D}}$, (ii) $Q_{\mathrm{cr} . n \mathrm{l}}^{1 \mathrm{D}}$, (iii) $Q_{\mathrm{cr} . \text { lin }}^{\mathrm{FEM}}$ and (iv) $Q_{\mathrm{cr} . n 1}^{\mathrm{FEM}}$. Fig. 14, on the other hand, shows a graphic representation of the critical load percentage increase due to the prebuckling deflections, calculated by means of $\Delta Q_{\mathrm{cr}}=$ $\left(Q_{\text {cr.nl }}-Q_{\text {cr.lin }}\right) / Q_{\text {cr.lin }} \times 100 \%$.

The analysis of these data prompts the following comments:

(i) Comparing the $Q_{\mathrm{cr} . l i n}^{1 \mathrm{D}}$ and $Q_{\mathrm{cr} \text {.lin }}^{\mathrm{FEM}}$ values, one observes the same general trend as in the cantilevers analysed previously. Indeed, with a single exception, all the relative errors are below $8 \%$. The exception concerns the prismatic cantilever acted by a centroidal load, for which the FEM linear critical load is about 20\% below its one-dimensional counterpart. As shown in Fig. $15 \mathrm{~b}$, this is due to the occurrence of a localised web buckling phenomenon, which does not take place when the load is applied at either one of the flanges - Figs. 15a-c. ${ }^{9}$

\footnotetext{
${ }^{9}$ When the load is applied at the top flange, this localised web buckling phenomenon does not occur because the LTB load is much smaller than the one concerning the centroidal load case (about $40 \%$ ).
}

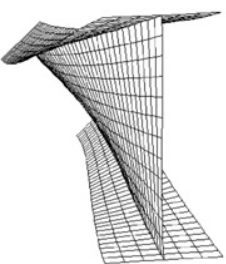

(a)

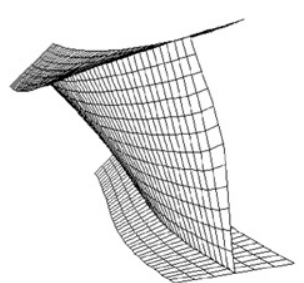

(b)

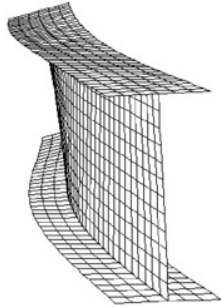

(c)
Fig. 15. Prismatic cantilevers (Fig. 3f): FEM-based critical mode shapes for tip load applied at the (a) top flange, (b) centroid and (c) bottom flange ( $L=4.0 \mathrm{~m}$ and linear buckling analysis).

(ii) With the same single exception, the $Q_{\text {cr.nl }}^{1 \mathrm{D}}$ values also never overestimate the $Q_{\mathrm{cr} . n \mathrm{l}}^{\mathrm{FEM}}$ ones by more than $8 \%$. Moreover, it is fair to say that the differences between the non-linear LTB critical loads yielded by the onedimensional and FE models are similar to those separating the linear ones, both for the prismatic and web-tapered cantilevers. Thus, it is not surprising that the critical load percentage increases given in Fig. 14 are also quite close (once again, with the exception of the prismatic cantilever acted by a centroidal load). 
(iii) Fig. 14 shows that, at least for the geometry and loading considered here, the effect of the pre-buckling deflections on the critical load is more relevant for the web-tapered cantilevers acted by loads applied at the centroid and bottom flange $\left(\Delta Q_{\mathrm{cr}} \approx 8 \%\right)$.

\section{Concluding remarks}

This paper presented and discussed the results of a comparative study carried out in order to assess the performance of a one-dimensional model of the elastic LTB behaviour of singly symmetric tapered thin-walled open beams, which may take into account the pre-buckling deflections. This study involved the critical loads and buckling modes of several prismatic and linearly web-tapered Isection cantilevers and simply supported beams, with equal or unequal uniform flanges, acted by point loads applied at various locations of the free end (cantilevers) or mid-span section (simply supported beams). These results were obtained from buckling analyses based on (i) the above one-dimensional model, which was numerically implemented by means of the Rayleigh-Ritz method, and (ii) two-dimensional shell finite element modelling, using the commercial code ABAQUS - the latter were taken as reference. Some of the FEA included rigid transverse web stiffeners located at a variable number of beam cross-sections. These stiffeners made it possible to restrain (at least partially) the cross-section distortion, thus simulating more closely one of the assumptions underlying the one-dimensional model.

On the basis of this comparative study, it was possible to conclude that, as long as the beams are not too short, the one-dimensional analyses yield reasonably accurate estimates of the critical loads and buckling mode shapes, regardless of whether the pre-buckling deflections are taken into account or not. Moreover, the accuracy of these estimates gradually increases with the beam length, a trend that reflects the decreasing relevance of the cross-section distortion (in-plane deformation). As for the considerable differences between the one-dimensional and shell FE models recorded for the shorter beams, it was found that they are mainly due to either (i) significant web and/or flange distortion or (ii) a localised web buckling phenomenon, which occurs in the neighbourhood of the load point of application - obviously, none of these phenomena can be captured by the one-dimensional model. This last assertion was corroborated by the FEM-based results obtained for beams with rigid transverse web stiffeners, as the presence of these stiffeners "pulled" the FEM-based critical loads towards the ones yielded by the one-dimensional model. Indeed, the larger gaps, associated with the shorter beams, were either completely (prismatic beams) or partially (tapered beams) closed. In the latter case, the distortion of the compressed flange appears to be responsible for the remaining differences - recall that no flange stiffening was ever incorporated in the FE analyses.

\section{Appendix A. List of symbols}

Upper case symbols

A undeformed configuration of a cross-section

$\boldsymbol{A}_{\mathrm{I}}, \boldsymbol{A}_{\mathrm{II}}$ orthonormal vectors spanning the plane tangent to $\mathrm{S}$

B undeformed configuration of the beam

C segment of $\mathrm{L}$ comprised between the point defined by $\theta^{2}=0$ and the point under consideration

$E \quad$ Young's modulus

$\bar{E}_{\alpha \beta} \quad$ covariant components of the Green-St. Venant membrane strain tensor

$A^{*}, S_{y}^{*}, z_{G}^{*}, I_{y}^{*}, I_{z}^{*}, I_{\rho}^{*}, I_{\omega}^{*}, I_{\omega z}^{*}, I_{\psi}^{*}, I_{\psi z}^{*}, I_{\omega \psi}^{*}, \beta_{y}^{*}$ geometrical properties of the undeformed beam, defined in Eq. (22)

$J \quad$ torsion constant of a cross-section

$L \quad$ length

$\mathrm{L}$ undeformed configuration of a cross-section midline

$M_{y}^{\mathrm{f}} \quad$ bending moment distribution in a fundamental state

$Q \quad$ point load

$\mathrm{S}$ undeformed configuration of the beam mid-surface

$\mathrm{U} \quad$ elastic strain energy

$U, V, W, \Phi$ generalised displacements in the one-dimensional model

$\bar{U}, \bar{V}, \bar{W}$ Cartesian components of the mid-surface displacement field

$V_{e} \quad$ potential energy of the external loads

$\Delta \quad$ relative error, taking as reference the results of the shell finite element analysis

$\Delta Q_{\mathrm{cr}}$ percentage increase in critical load due to the prebuckling deflections

$\Pi \quad$ total potential energy of the beam-load system

Lower case symbols

a determinant of $a_{\alpha \beta}$

$\boldsymbol{a}_{1}, \boldsymbol{a}_{2}\left(\boldsymbol{a}^{1}, \boldsymbol{a}^{2}\right)$ covariant (contravariant) base vectors of the plane tangent to $\mathrm{S}$

$a_{\alpha \beta}\left(a^{\alpha \beta}\right)$ covariant (contravariant) components of the metric tensor of $\mathrm{S}$ (first fundamental form of $\mathrm{S}$ )

$a^{\alpha \beta \chi \delta} \quad$ contravariant components of the two-dimensional elasticity tensor of the membrane shell

$h$ height of an I-section, measured between the flange mid-lines

$\bar{n}^{\alpha \beta} \quad$ contravariant components of the membrane force (or stress resultant) tensor

$\bar{r} \quad$ position vector of a point in $\mathrm{S}$ (relative to the origin of the fixed Cartesian frame)

$t \quad$ wall thickness

$t^{*} \quad$ "reduced" wall thickness, equal to $t a^{-3 / 2}$

$u, v, w, \phi$ kinematically admissible variations of the generalised displacements

$x, y, z$ Cartesian coordinates

$\alpha \quad$ ratio between the minimum and maximum heights of a web-tapered I-beam

$\delta^{2}($.$) \quad second variation of a function or functional$ 
$\lambda \quad$ load factor

$v \quad$ Poisson's ratio

$\bar{\omega} \quad$ sectorial coordinate

$\psi \quad$ tapering function (i.e. directly associated with the cross-section variation)

$\theta^{1}, \theta^{2} \quad$ Gaussian coordinates on $\mathrm{S}$

\section{Subscripts, superscripts and other symbols}

(.) $)_{\mathrm{cr}}$ critical value

(.) cr.lin critical value obtained from a linear buckling analysis (pre-buckling deflections disregarded)

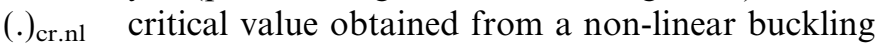
analysis (pre-buckling deflections accounted for)

(.) ${ }^{\mathrm{f}} \quad$ quantity associated with the fundamental equilibrium path

(.) ${ }^{\text {FEM }}$ result obtained from a shell finite element analysis

(.) $)^{1 \mathrm{D}}$ result obtained from a one-dimensional analysis

(.),$\alpha \quad$ derivative with respect to $\theta^{\alpha}$

$\left\{O ; \boldsymbol{e}_{1}, \boldsymbol{e}_{2}, \boldsymbol{e}_{3}\right\}$ fixed orthonormal right-handed Cartesian frame

$\|$.$\| \quad Euclidean norm in \mathbf{R}^{3}$

. $\quad$ Euclidean inner product in $\mathbf{R}^{3}$

$\times \quad$ cross product in $\mathbf{R}^{3}$

\section{References}

[1] Comité Européen de Normalisation (CEN). Eurocode 3: Design of steel structures. Part 1-1: General rules and rules for buildings (EN 1993-1-1). Brussels; 2005.

[2] Comité Européen de Normalisation (CEN). Eurocode 3: Design of steel structures. Part 1-1: General rules and rules for buildings (ENV 1993-1-1). Brussels; 1992.

[3] Andrade A, Camotim D. Lateral-torsional buckling of singly symmetric tapered beams: theory and applications. J Eng Mech (ASCE) 2005;131(6):586-97.

[4] Vlassov B. Thin-walled elastic bars. Jerusalem: Israel Program for Scientific Translations; 1961.

[5] Andrade A. Lateral-torsional buckling of tapered beams. M.Sc. Thesis, Department of Civil Engineering, Technical University of Lisbon; 2003 [in Portuguese].

[6] Andrade A, Camotim D. Lateral-torsional buckling of prismatic and tapered thin-walled open beams: assessing the influence of prebuckling deflections. Steel Compos Struct 2004;4(4):281-301.

[7] Yang Y, Yau J. Stability of beams with tapered I-sections. J Eng Mech (ASCE) 1987;113(9):1337-57.

[8] Bradford M, Cuk P. Elastic buckling of tapered monosymmetric I-beams. J Struct Eng (ASCE) 1988;114(5):977-96.
[9] Ronagh H, Bradford M, Attard M. Nonlinear analysis of thin-walled members of variable cross-section Part II: application. Comput Struct 2000;77(3):301-13.

[10] Boissonnade N, Muzeau JP. New beam finite element for tapered members. In: Topping B, editor. Proceedings of the 8th international conference on civil and structural engineering computing. Stirling: Civil-Comp Press; 2001. p. 73-4 [CD-ROM paper \# 27].

[11] Pi Y, Trahair NS. Prebuckling deflections and lateral buckling. Part II: applications. J Struct Eng (ASCE) 1992;118(11):2967-85.

[12] Hibbit, Karlsson and Sorensen Inc. Abaqus standard (version 6.3); 2002.

[13] Polyzois D, Qing L. Stability of web-tapered beams. In: Proceedings of the structural stability research council annual technical session and meeting; 1993. p. 179-92.

[14] Polyzois D, Raftoyiannis I. Lateral-torsional stability of steel webtapered I-beams. J Struct Eng (ASCE) 1998;124(10):1208-16.

[15] Ronagh H, Bradford M. A rational model for the distortional buckling of tapered members. Comput Meth Appl Mech Eng 1996; 130(3-4):263-77.

[16] Braham M, Heck C. Tests and numerical simulation on lateraltorsional buckling of tapered I-section beams. In: Iványi M, editor. Stability and ductility of steel structures. Budapest: Akadémia Kiadó; 2002.

[17] European Commission for Steel and Coal (ECSC). Lateral-torsional buckling in steel and composite beams. Research project 7210-PR183 final technical report (Book 3); 2003.

[18] Antman SS. The theory of rods. In: Truesdell C, editor. Encyclopaedia of physics, vol. VIa/2. Berlin: Springer; 1972. p. 641-703.

[19] Ciarlet PG. Mathematical elasticity. Theory of shells, vol. 3. Amsterdam: Elsevier; 2000

[20] Ciarlet PG. Mathematical elasticity. Three-dimensional elasticity, vol. 1. Amsterdam: Elsevier; 1988.

[21] Lee G, Szabo B. Torsional response of tapered I-girders. J Struct Div (ASCE) 1967;93(5):233-52.

[22] Necas J, Hlavácek I. Mathematical theory of elastic and elasto-plastic bodies: an introduction. Amsterdam: Elsevier; 1981.

[23] Bazant Z, Cedolin L. Stability of structures - elastic, inelastic, fracture and damage theories. New York: Oxford University Press; 1991.

[24] Mikhlin SG. Variational methods in mathematical physics. Oxford: Pergamon Press; 1964.

[25] Dinis PB, Camotim D. Local-plate and distortional post-buckling behavior of cold-formed steel columns: elastic and elastic-plastic FEM analysis. In: Proceedings of structural stability research council annual stability conference; 2004. p. 475-98.

[26] Dinis PB, Camotim D. Stability of cold-formed steel members: finite element modelling and assessment of the influence of the support conditions. In: Barbosa JL, editor. Proceedings of the VII congress on applied and computational mechanics; 2003. p. 365-77 [in Portuguese].

[27] Bathe KJ. Finite element procedures. New Jersey: Prentice-Hall; 1998. 